\title{
Ecogenomics and biogeochemical impacts of uncultivated globally abundant ocean viruses
}

Simon Roux ${ }^{1}$, Jennifer R. Brum ${ }^{1}$, Bas E. Dutilh ${ }^{2,3,4}$, Shinichi Sunagawa ${ }^{5}$, Melissa B. Duhaime ${ }^{6}$, Alexander Loy ${ }^{7,8}$, Bonnie T. Poulos ${ }^{9}$, Natalie Solonenko ${ }^{1}$, Elena Lara ${ }^{10,11}$, Julie Poulain ${ }^{12}$, Stéphane Pesant ${ }^{13,14}$, Stefanie Kandels-Lewis ${ }^{5,15}$, Céline Dimier ${ }^{16}$, Marc Picheral ${ }^{17}$, Sarah Searson ${ }^{17,18}$, Corinne Cruaud $^{12}$, Adriana Alberti ${ }^{12}$, Carlos M. Duarte ${ }^{19,20}$, Josep M. Gasol ${ }^{10}$, Dolors Vaqué ${ }^{10}$, Tara Oceans Coordinators ${ }^{\dagger}$, Peer Bork ${ }^{5,21}$, Silvia G. Acinas ${ }^{10}$, Patrick Wincker ${ }^{12,22,23}$, Matthew B. Sullivan ${ }^{1,24}$ *

10

${ }^{1}$ Department of Microbiology, The Ohio State University, Columbus, OH, USA

${ }^{2}$ Theoretical Biology and Bioinformatics, Utrecht University, Utrecht, The Netherlands.

${ }^{3}$ Centre for Molecular and Biomolecular Informatics, Radboud University Medical Centre, Nijmegen, The Netherlands.

${ }^{4}$ Department of Marine Biology, Federal University of Rio de Janeiro, Rio de Janeiro, Brazil

$15{ }^{5}$ Structural and Computational Biology, European Molecular Biology Laboratory, Heidelberg, Germany

${ }^{6}$ Department of Ecology and Evolutionary Biology, University of Michigan, MI, USA

${ }^{7}$ Division of Microbial Ecology, Department of Microbiology and Ecosystem Science, Research Network Chemistry Meets Microbiology, University of Vienna, Vienna, Austria

${ }^{8}$ Austrian Polar Research Institute, Vienna, Austria

$20{ }^{9}$ Department of Ecology and Evolutionary Biology, University of Arizona, Tucson, AZ, USA

${ }^{10}$ Department of Marine Biology and Oceanography, Institut de Ciències del Mar (CSIC), Barcelona, Spain

${ }^{11}$ Institute of Marine Sciences (CNR-ISMAR), National Research Council, Venezia, Italy

${ }^{12}$ CEA - Institut de Génomique, GENOSCOPE, Evry, France

${ }^{13}$ PANGAEA, Data Publisher for Earth and Environmental Science, University of Bremen, Bremen, Germany

${ }^{33}$ MARUM, Bremen University, Bremen, Germany

${ }^{15}$ Directors' Research European Molecular Biology Laboratory, Heidelberg, Germany

${ }^{16}$ Sorbonne Universités, UPMC Université Paris 06, CNRS, Laboratoire Adaptation et Diversité en Milieu Marin, Station

Biologique de Roscoff, Roscoff, France

${ }^{17}$ Sorbonne Universités, UPMC Université Paris 06, CNRS, Laboratoire d'oceanographie de Villefranche (LOV),

30 Observatoire Océanologique, Villefranche-sur-Mer, France

${ }^{18}$ Department of Oceanography, University of Hawaii, Honolulu, Hawaii, USA

${ }^{19}$ Mediterranean Institute of Advanced Studies, CSIC-UiB, Esporles, Mallorca, Spain

${ }^{20}$ King Abdullah University of Science and Technology (KAUST), Red Sea Research Center (RSRC), Thuwal, Saudi

Arabia

$35 \quad{ }^{21}$ Max-Delbrück-Centre for Molecular Medicine, 13092 Berlin, Germany

${ }^{22}$ CNRS, UMR 8030, CP5706, Evry, France

${ }^{23}$ Université d'Evry, UMR 8030, CP5706, Evry, France

${ }^{24}$ Department of Civil, Environmental and Geodetic Engineering, The Ohio State University, Columbus, OH, USA

$40 \dagger$ Tara Oceans coordinators and affiliations are listed following the Acknowledgements.

*correspondence to mbsulli@gmail.com 


\section{Abstract}

Ocean microbes drive global-scale biogeochemical cycling ${ }^{1}$, but do so under constraints imposed by and cultivation challenges, genome-level viral diversity remains poorly described and grossly understudied in nature such that $<1 \%$ of observed surface ocean viruses, even those that are abundant and ubiquitous, are 'known'5. Here we analyze a global map of abundant, double stranded DNA (dsDNA) viruses and viral-encoded auxiliary metabolic genes (AMGs) with genomic and ecological

50 contexts through the Global Ocean Viromes (GOV) dataset, which includes complete genomes and large genomic fragments from both surface and deep ocean viruses sampled during the Tara Oceans and Malaspina research expeditions ${ }^{6,7}$. A total of 15,222 epi- and mesopelagic viral populations were identified that comprised 867 viral clusters (VCs, approximately genus-level groups ${ }^{8,9}$ ). This roughly triples known ocean viral populations ${ }^{10}$, doubles known candidate bacterial and archaeal virus genera ${ }^{9}$,

55 and near-completely samples epipelagic communities at both the population and VC level. Thirty-eight of the 867 VCs were identified as the most impactful dsDNA viral groups in the oceans, as these were locally or globally abundant and accounted together for nearly half of the viral populations in any GOV sample. Most of these were predicted in silico to infect dominant, ecologically relevant microbes, while two thirds of them represent newly described viruses that lacked any cultivated representative. Beyond 60 these taxon-specific ecological observations, we identified 243 viral-encoded AMGs in GOV, only 95 of which were known. Deeper analyses of 4 of these AMGs revealed that abundant viruses directly manipulate sulfur and nitrogen cycling, and do so throughout the epipelagic ocean. Together these data provide a critically-needed organismal catalog and functional context to begin meaningfully integrating viruses into ecosystem models as key players in nutrient cycling and trophic networks.

\section{Main text}

The fundamental bottleneck preventing the incorporation of viruses of microbes into predictive ecosystem models is the lack of quantitative surveys of viral diversity in nature. This is because (i) most naturally-occurring microbes and viruses resist being cultured, and (ii) viruses lack a universally

70 conserved marker gene, which precludes barcode surveys of uncultivated viral diversity ${ }^{5}$. While viral metagenomics was introduced to circumvent these issues, low-throughput sequencing technologies initially yielded highly fragmented datasets suitable only for strongly database-biased descriptions ${ }^{11}$, and gene-level analyses of environmental viral communities (reviewed in ref. 5).

Subsequent improvements in experimental methods, sequencing technologies, and analytical

75 approaches progressively enabled viral population ecology through the availability of genomic information $^{5,12-14}$. For example, 1,148 large viral genome fragments captured in a fosmid library of Mediterranean Sea microbes revealed remarkable viral diversity in a single sample, with some of these genomes seemingly globally distributed based upon the six viral metagenomic datasets available at the time $^{12}$. Similarly, 69 viral genomes assembled from single-cell genomic datasets provided reference

80 genomes that illuminated the ecology, evolution and biogeochemical impacts of viruses infecting an uncultivated anaerobic chemoautotroph ${ }^{14}$. Beyond these 'omics-enabled experimental advances, metagenomic approaches have matured to be quantitative ${ }^{5}$ and informative enough, at least for dsDNA templates, to themselves provide genomic information on viruses that infect both abundant and rare hosts. For example, the analysis of 43 surface ocean viral metagenomes (viromes) comprising the Tara

85 Oceans Viromes (TOV) dataset revealed the global underlying structure of these communities, and identified 5,476 viral populations, only 39 of which were previously known ${ }^{10}$.

Here we further identify ocean viral populations, characterize the most abundant and widespread types of ocean viruses, and analyze new viral-encoded AMGs and their distributions to expand our understanding of how viruses modulate microbial biogeochemistry. We do so on the basis of a new

90 Global Oceans Viromes (GOV) dataset, which augments TOV with 61 new samples to better represent the surface and deep oceans, and now totals 104 ocean viromes representing $925 \mathrm{Gbp}$ of sequencing 
(Supplementary Table 1). Beyond better sample coverage, analytical approaches including cross-assembly $^{15}$ and genome binning ${ }^{16}$ make the GOV dataset a much improved genomic representation of the sampled viruses. From 1,380,834 contigs generated, which recruited $67 \%$ of the

95 reads, we identified 15,280 viral populations (Fig. 1A, Supplementary Fig. 1). This expands the number of known ocean viral populations nearly 3 -fold over the prior TOV dataset ${ }^{10}$, while also improving the genomic context for these TOV-known populations by a 2.5 -fold increase in contig length on average (Supplementary Table 2). Rarefaction analyses show that while mesopelagic viral communities remain undersampled, epipelagic viral communities now appear near-completely sampled (Extended Data Fig.

100 1A). Because bathypelagic communities were underrepresented due to cellular contamination of these viromes, we focused the remaining analyses on the 15,222 non-bathypelagic viral populations.

We first categorized new and known viral populations into viral clusters, or VCs (Supplementary Fig. 1) using shared gene content information and network analytics ${ }^{8}$. This method starts from genome fragments $(\geq 10 \mathrm{~kb})$ and results in VCs approximately equivalent to known bacterial and archaeal virus 105 genera $^{8,9}$ (see also Supplementary Text, Extended Data Fig. 2 \& Supplementary Table 3 for comparison with alternative classification methods). Combining the 15,222 viral populations identified here with the genomes and genome fragments of another 15,929 publicly available bacterial and archaeal viruses generated a total of 1,259 VCs. Of these, 658 included exclusively GOV sequences, which approximately doubles known bacterial and archaeal virus genera ${ }^{9}$, and another 209 VCs contained at $^{2}$

110 least one GOV sequence (Fig. 1B). As with viral populations, rarefaction analyses suggested that VC diversity was undersampled in mesopelagic waters, but near-completely sampled in epipelagic waters (Extended Data Fig. 1B).

We next identified the most abundant and widespread VCs based on read recruitment of VC members. In each sample, a fraction of the VCs were identified as abundant based on their cumulative 115 contribution to sample diversity (estimated with the Simpson Index, abundant VCs represent $80 \%$ of the total sample diversity, Extended Data Fig. 1C). By these criteria, only 38 of the 867 VCs observed were abundant in two or more stations, and together recruited an average of $50 \%$ and $35 \%$ of the reads from viral populations for epi- and mesopelagic samples, respectively (Supplementary Table 3). Four of these 38 abundant VCs were also relatively ubiquitous as they were abundant in more than 25 stations, 120 and 62 of the 91 non-bathypelagic samples were dominated by 1 of these 4 VCs (Fig. 2 A \& B). Among the 38 abundant VCs, only 2 corresponded to well-studied viruses, from the T4 superfamily ${ }^{17,18}$ (VC_2, 1 of the 4 ubiquitous) and the T7virus genus ${ }^{19}$ (VC_9), whereas 8 represented known, but unclassified viral isolates, another 10 included viruses previously only identified in environmental libraries ${ }^{12,13}$, and the remaining 18 VCs were completely novel (Fig. 2C, Extended Data Fig. 3).

125 Given this global map of the dominant dsDNA viral types in the oceans, we next sought to identify the range of hosts that these viruses infect. Large-scale host range estimations are challenging as culture-based methods experimentally link viruses and hosts, but insufficiently capture naturally-occurring diversity, whereas metagenomic approaches broadly survey diversity, but struggle to establish virus-host linkages. However, recent work has demonstrated the predictive power of

130 sequence-based approaches such as similarities between (i) viral genomes and host CRISPR spacers ${ }^{20}$ (ii) viral and microbial genomes due to integrated prophages or gene transfers ${ }^{12}$ and (iii) viral and host genome nucleotide signatures (here, tetranucleotide frequencies) ${ }^{9}$. We applied all 3 methods to the GOV dataset and predicted hosts at the phylum level, or class level for Proteobacteria (Supplementary Table 4). These results were then summarized at the VC level, leading to host range predictions for 392

135 of $867 \mathrm{VCs}$, and for which confidence was assessed by comparison to a null model (Supplementary Fig. 1).

The hosts of the 38 globally abundant VCs were largely restricted to abundant and widespread epipelagic-ocean microbes identified from mi Tag-based OTU counts in Tara Oceans microbial metagenomes ${ }^{21}$. Notably, the 4 ubiquitous and abundant VCs were predicted to infect 7 of the 8 globally abundant microbial groups (Actinobacteria, Alpha-, Delta-, and Gamma-proteobacteria, 
Bacteroidetes, Cyanobacteria, Deferribacteres; Fig. 2C, Extended Data Fig. 4). The $8^{\text {th }}$ abundant microbial group, Euryarchaeota, was not linked to these $4 \mathrm{VCs}$, but was predicted as a host for 2 of the 34 other abundant VCs (VC_3 and VC_63, Extended Data Fig. 3). Among the 38 abundant VCs, the number of VCs predicted to infect a given microbial host phylum (or class for Proteobacteria) was positively correlated with host global richness rather than abundance (Extended Data Fig. 4B). This suggests that, likely because of the global distribution of ocean viruses ${ }^{10,22}$, widespread and abundant hosts that are minimally diverse (e.g. Cyanobacteria) provide few viral niches, whereas more diverse host groups, even at lower abundance (e.g. Betaproteobacteria), provide more opportunity for viral niche differentiation. These data thus provide critically-needed empirical support for guiding and testing hypotheses derived from global virus-host network models ${ }^{23}$.

Having mapped viral diversity and predicted virus-host pairings, we next sought to identify novel virus-encoded auxiliary metabolic genes, or AMGs, that might modify host metabolism during infection. To maximize the detection of $\mathrm{AMG}$, all viral contigs $>1.5 \mathrm{~kb}$ were examined, including small contigs not associated with a viral population, which totaled 298,383 contigs. This revealed 243 putative AMGs (Supplementary Table 5). While 95 were already known (reviewed in ref. 24), others offer new insights into how viruses directly manipulate microbial metabolisms beyond photosynthesis and carbon metabolism ${ }^{25-28}$. Here we focus specifically on 4 AMGs $(d s r C$, sox YZ, P-II and amoC, see Extended Data Table 1) because of their critical roles in sulfur or nitrogen cycling and their novelty in epipelagic ocean viruses. Three of these are not yet known in viruses, and one, $d s r C$-like genes, has been observed in viruses, but only from anoxic deep-sea environments ${ }^{14,29}$.

Sulfur oxidation in seawater involves two central microbial pathways - Dsr and Sox ${ }^{30}$ - and GOV AMG analyses revealed that epipelagic viruses encode key genes for each. First, $11 d s r C$-like genes were identified in viral contigs (Extended Data Fig. 5). The Dsr operon is used by sulfate/sulfitereducing microbes in anoxic environments, as well as sulfur-oxidizing bacteria in oxic and anoxic

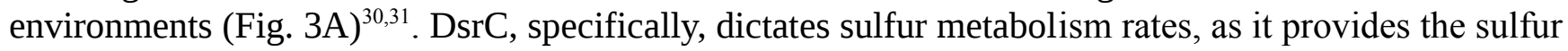
substrate to DsrAB-sulfite reductase for processing ${ }^{32}$. A conserved C-terminal motif with two cysteine residues $\mathrm{Cys}_{\mathrm{B}} \mathrm{X}_{10} \mathrm{Cys}_{\mathrm{A}}$ is essential for this function. Outside of energy metabolism, a second class of $d s r \mathrm{C}$-like genes (also known as $t u s \mathrm{E}$ ) lack $\mathrm{Cys}_{\mathrm{B}}$ and are instead involved in tRNA modification ${ }^{33}$. In the GOV dataset, four clades of $d s r C$-like sequences were similar to TusE (DsrC-1 to DsrC-4), whereas the 170 fifth (DsrC-5) was most similar to bona fide DsrC involved in sulfur oxidation (Extended Data Fig. 5, Extended Data Table 1, Supplementary Fig. 2). Second, 4 soxYZ genes from the sox operon were identified on viral contigs (Extended Data Fig. 6) ${ }^{30,31}$. Like DsrC, SoxYZ is an important sulfur carrier protein harboring a sulfur interaction motif identified in GOV SoxYZ proteins (Fig. 3A, Supplementary Fig. 3) ${ }^{34}$.

175 Complementarily, GOV AMG analyses suggested that viruses also manipulate nitrogen cycling. First, $10 \mathrm{GOV}$ contigs encoded P-II, a nitrogen metabolism regulator that is widespread across bacteria and archaea (Fig. 3B $)^{35,36}$. Functional P-II genes contain a conserved C-terminal motif, and a conserved $\mathrm{Y}$ residue that is uridylylated under nitrogen-limiting conditions ${ }^{35}$. One AMG clade (P-II-3) lacked this conversed $\mathrm{Y}$ residue leaving its function ambiguous, whereas 3 clades (P-II-1, P-II-2, and P-II-4)

180 displayed both conserved motifs and were also predicted to have structures similar to bona fide P-II, which suggests these AMGs are functional (Supplementary Fig. 4). Second, two of these P-II AMG clades (P-II-1 and P-II-4) were proximal to an ammonium transporter gene, amt, in viral contigs (Extended Data Fig. 7). In bacteria, such an arrangement is a signature of P-II-like genes that specifically activate alternative nitrogen production and ammonia uptake pathways during nitrogen 185 starvation $^{35,36}$. Third, one GOV contig included amoC, the gene coding for the subunit $\mathrm{C}$ of ammonia monooxygenase, suggesting a role in ammonia oxidation ${ }^{37,38}$. While functional annotation is challenging for these genes ${ }^{38}$, and functional motifs are not yet available, the translated AMG was 94\% identical to functional AmoC from Thaumarchaeota (Extended Data Fig. 8, Supplementary Fig. 5). Such exceptionally high identity is rarely observed among AMGs, and compars only to the well-studied 
190 PsbA genes, which are expressed and functional ${ }^{39}$. We posit that this highly conserved amoC AMG is also functional.

Next, we investigated the origin, evolutionary history, and diversity of these AMGs in epipelagic viruses. The $15 \mathrm{GOV}$ contigs encoding $d s r C$ or $\operatorname{sox} Y Z$ genes were all affiliated to T4 superfamily contigs, one of the 4 abundant and ubiquitous VCs (VC_2, Extended Data Fig. 5 and 6, Extended Data

195 Table 1). Both DsrC and Sox phylogenies suggested that these viruses obtained each AMG only once from probable S-oxidizing proteobacterial hosts (Extended Data Fig. 5 and 6). Among the latter, DsrC-5, the bona fide S-oxidation DsrC, appeared most closely related to a clade of uncultivated S-oxidizing Gammaproteobacteria represented by bacterial artificial chromosome MED13k09 (confirmed by phylogenetic analyses of the sulfur oxidizer marker DsrA, Supplementary Fig. 6). If

200 DsrC-5-containing viruses indeed infect members of this clade, they would impact bacteria that are widespread in the epipelagic ocean ${ }^{40}$, and suspected to degrade dimethyl sulfide, the most important reduced sulfur species in oxygenated ocean waters and key compound in the transport of sulfur from ocean to atmosphere and in cloud formation ${ }^{41}$. In contrast to the sulfur AMGs, phylogenies suggest that P-II AMGs originated from diverse viruses (6 VCs including the abundant VC_2 and VC_12), and 205 were acquired at least 4 times independently from different host phyla (Bacteroidetes, Proteobacteria, and possibly Verrucomicrobia, Extended Data Fig. 7). Finally, while a single amoC AMG offers only preliminary evaluation of its evolutionary history, this amoC-encoding contig appears to represent novel and rare unclassified archaeal dsDNA viruses (VC_623), which presumably infect ammoniaoxidizing Thaumarchaeota, known for their major role in global nitrification ${ }^{37}$ (Extended Data Fig. 8).

210 Finally, we investigated the ecology of viruses encoding these new AMGs by mapping their distribution across the GOV dataset. Seven AMG clades were geographically restricted (DsrC-unc, DsrC-1, DsrC-2, DsrC-4, P-II-2, P-II-3, and amoC), and 5 were widespread throughout the epipelagic (DsrC-3, DsrC-5, SoxYZ, P-II-1) or mesopelagic (P-II-4) ocean (Fig. 3C). While all widespread epipelagic AMGs were detected in waters of mid-range temperatures, DsrC-5 and SoxYZ were 215 predominantly detected in low nutrients conditions (low phosphate and nitrite), while P-II-1 was predominantly detected in high nutrient conditions (high phosphate, nitrate and nitrite, Fig. 3D, Extended Data Fig. 9). Thus, we hypothesize that viruses utilize DsrC-5 or SoxYZ to boost sulfur oxidation rates when infecting sulfur oxidizers in low-nutrient conditions, and P-II under high-nutrient conditions favorable for normal host growth. The latter could be particularly useful to viruses by

220 activating expensive and stress-inducing alternative N-producing pathways typically only used under $\mathrm{N}$-starvation conditions ${ }^{35,36}$. Consistent with this, metatranscriptomes from three low-nutrient stations (11_SRF in Mediterranean Sea, 39_DCM in Arabian Sea, and 151_SRF in Atlantic Ocean) revealed expression of viral homologs of $d s r \overline{\mathrm{C}}$ and sox YZ but not of viral P-II (Extended Data Table 1).

Overall, this systematically collected and processed GOV dataset brings critically-needed and

225 unprecedented global ecological context to new and known, surface and deep ocean viruses. Global diversity analyses identified and mapped abundant dsDNA viruses at the population- and VC-level, and indicated that these are near-completely sampled in epipelagic waters and dominated by few viral groups, mostly newly described. The characterization of new viral-encoded AMGs, their viral carriers, possible impacted hosts, and biogeographical patterns revealed that viral manipulation of cellular 230 processes involves much more than photosynthesis and carbon metabolism ${ }^{25-28}$, to also now include nitrogen and sulfur cycling throughout the epipelagic ocean. These advances are foundational for interpretation of new (meta)genomic datasets and selection of relevant experimental systems to develop, and, together with myriad experimental, informatic and theoretical advances already occurring ${ }^{5,15,42-44}$, will accelerate the field towards understanding and predicting the roles and global 235 impacts of viruses in nature. 

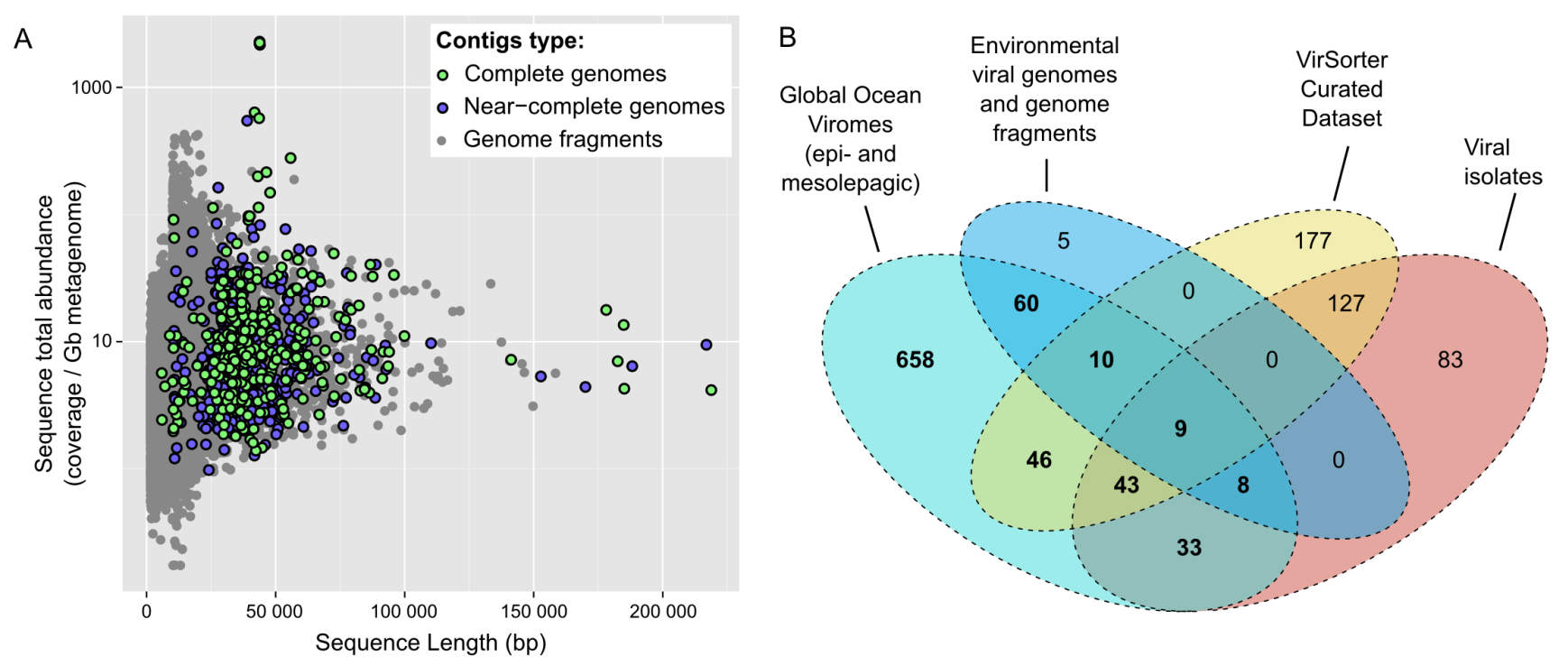

Figure 1: Composition and novelty of the Global Ocean Viromes (GOV) dataset. A. Size of viral contigs (x-axis) and cumulative coverage across the GOV dataset (y-axis). Contigs corresponding to complete or near-complete genomes (based on the size of similar complete genomes) are indicated. For clarity, only contigs associated with a viral population are displayed. B. Distribution of all viral clusters

240 (VCs) according to the origin of their members. Viral genomes (or fragments) in a VC can originate from isolate viral genomes, the VirSorter Curated Dataset ${ }^{9}$ (viral genomes identified in silico from microbial genomes), environmental viral genomes and genome fragments (e.g. from fosmid libraries), or the GOV dataset. VCs including at least one GOV sequence and further analyzed in this study are highlighted in bold. 


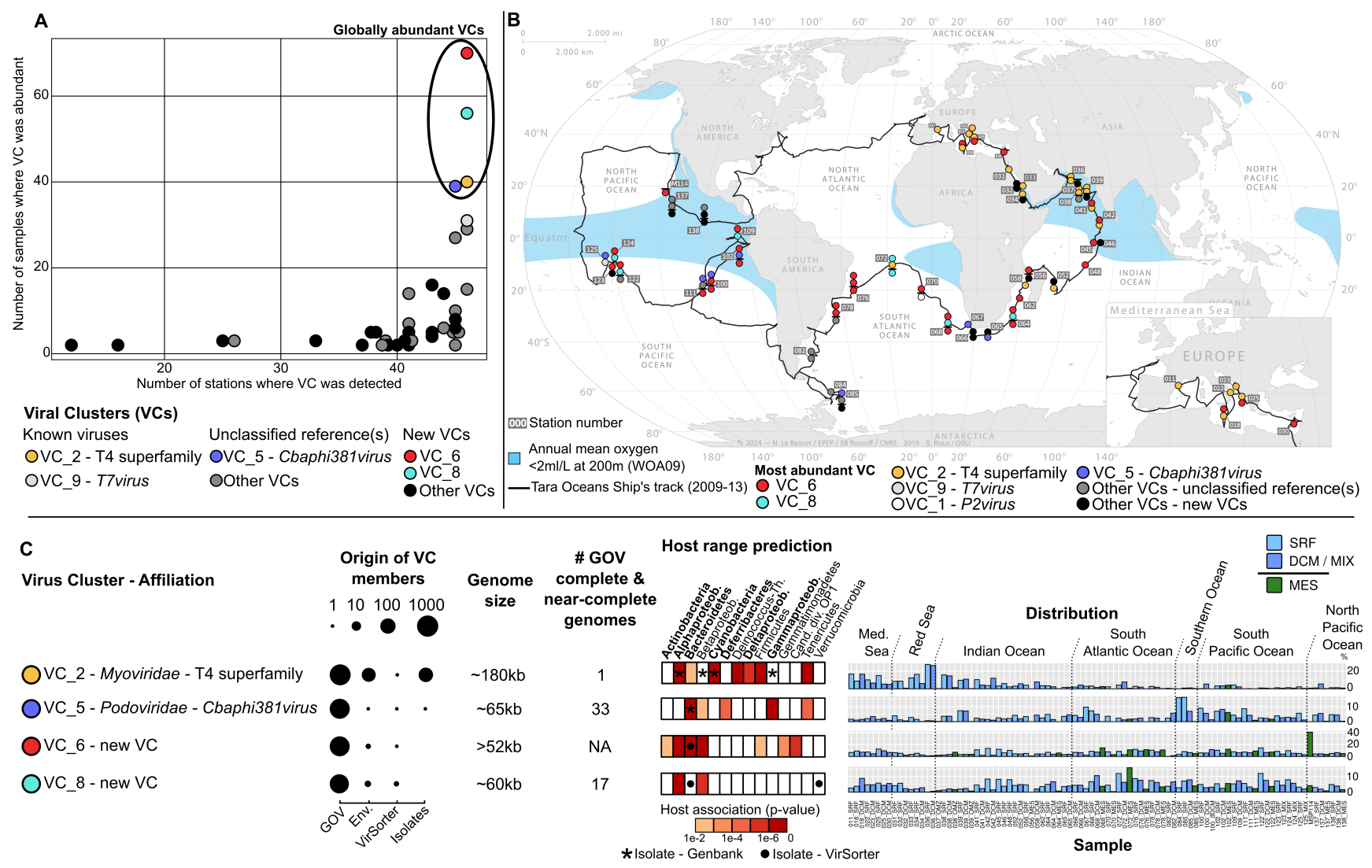

245 Figure 2: Characterization of the dominant oceanic viral clusters (VCs). A. Distribution and abundance of the 38 recurrently abundant VCs according to the total number of stations in which members of the VC were detected (x-axis), and the number of samples in which the VC was detected in the abundant fraction (y-axis). VCs are classified by degree of novelty: "Known viruses" are VCs corresponding to established viral groups in ICTV classification, "Unclassified reference(s)" are VCs including genomes from unclassified isolate(s), and "New VCs" are only composed of environmental viral sequences (i.e. no isolate). The 4 widespread and abundant VCs are highlighted with colored circles. B. GOV samples with their most abundant VC mapped to station locations. Samples are stacked vertically when multiple samples from different depths are available (a horizontal line is used to separate epipelagic from mesopelagic samples). C. Summary of VC affiliation, origin of the VC members (Env: environmental viral sequences), estimated genome size, predicted host range, and distribution of the 4 ubiquitous VCs circled in panel A (relative abundance are indicated as $\%$ of the viral populations identified). The abundant epipelagic microbial groups (representing $>1 \%$ of the microbial OTUs abundance of epipelagic samples) are highlighted in bold; Alphaproteob.Alphaproteobacteria, Betaproteob.-Betaproteobacteria, Deinococcus-Th.-Deinococcus-Thermus, 260 Deltaproteob.-Deltaproteobacteria, Gammaproteob.-Gammaproteobacteria, Cand div OP1-Candidate division OP1. Oceanic basins are indicated for VCs distributions; Med. Sea-Mediterranean Sea. 


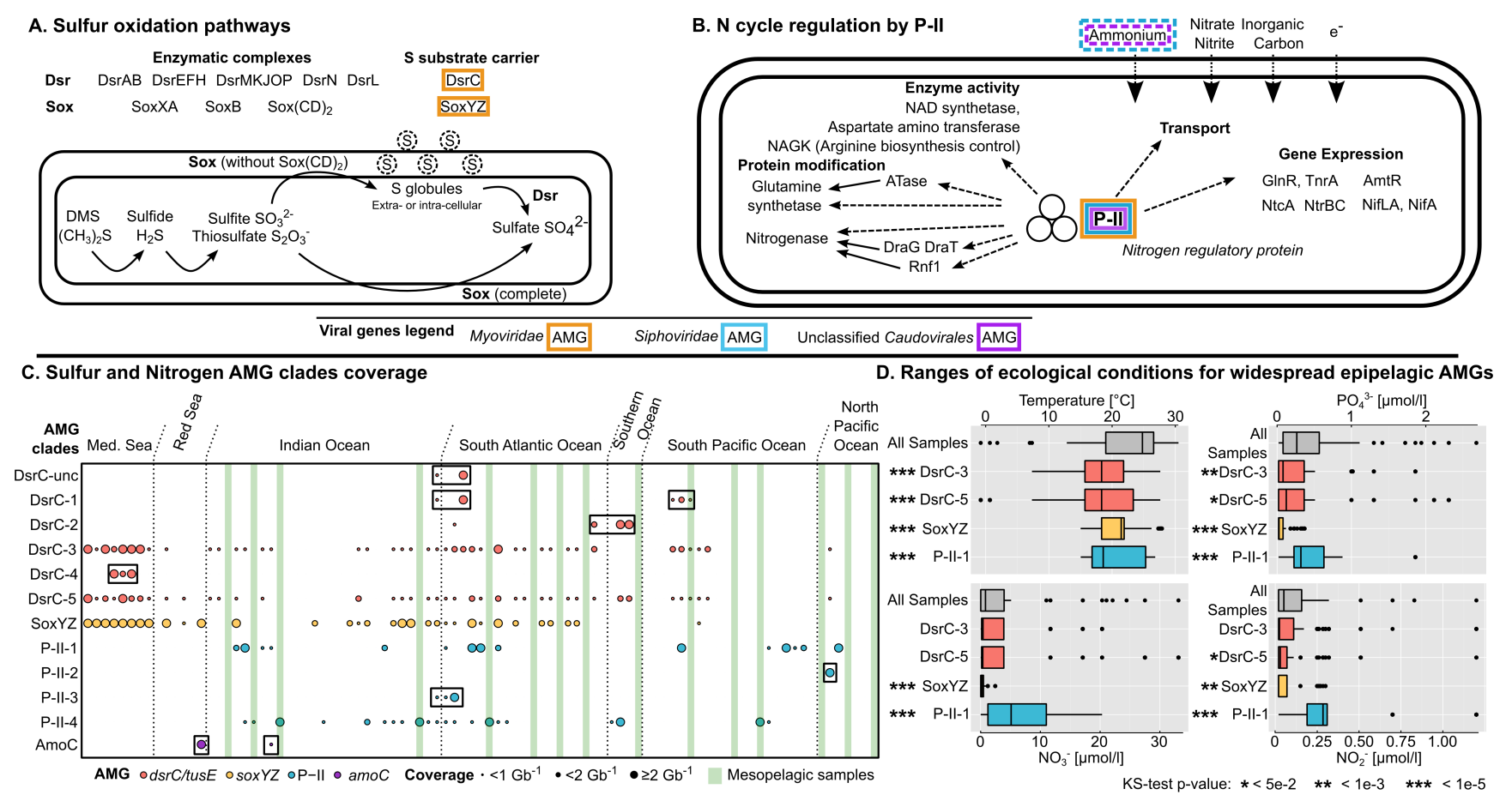

Figure 3: Characterization and distribution of viral Auxiliary Metabolic Genes (AMGs) involved

in sulfur and nitrogen cycles. Schematics are presented for (A) microbial sulfur oxidation pathways involving the two main gene clusters ( $d s r$ and $s o x)$ and (B) the central role of the P-II protein in cell regulation (adapted from ${ }^{35,45}$ ). AMGs are outlined in colors according to the taxonomic affiliation (family) of the corresponding virus. Ammonium transporters detected next to viral P-II are highlighted with a dashed outline. C. Distribution of viral AMG clades. Mesopelagic samples are highlighted in dark green, and clades with restricted geographic distributions are highlighted with black boxes. D. Temperature and nutrient conditions for which widespread epipelagic AMGs tend to be most abundant. For each environmental parameter, the range of values across all epipelagic samples is displayed as "All Samples" alongside distributions representing the range of values where each AMG clade was detected, weighted by the AMG coverage across these samples (see Extended Data Fig. 9 for underlying coverage data). Distributions significantly different from the "All Samples" distribution (two-sided KS-test) are indicated with stars. 


\section{Methods}

\section{Sample collection and processing}

\section{Tara Oceans expedition}

Ninety samples were collected between October 10, 2009, and December 12, 2011, at 45 locations throughout the world's oceans (Supplementary Table 1) through the Tara Oceans Expedition ${ }^{46}$. These included samples from a range of depths: surface, deep chlorophyll maximum, bottom of mixed layer when no deep chlorophyll maximum was observed (Station 123, 124, and 125), and mesopelagic

285 samples. The sampling stations were located in 7 oceans and seas, 4 different biomes and 14 Longhurst oceanographic provinces (Supplementary Table 1). For TARA station 100, two different peaks of chlorophyll were observed, so two samples were taken at the shallow (100_DCM) and deep (100_dDCM) chlorophyll maximum. For each sample, $20 \mathrm{~L}$ of seawater were $0 . \overline{2} 2 \mu \mathrm{m}$-filtered and viruses were concentrated from the filtrate using iron chloride flocculation ${ }^{47}$ followed by storage at $4^{\circ} \mathrm{C}$.

290 After resuspension in ascorbic-EDTA buffer (0.1 M EDTA, 0.2 M Mg, 0.2 M ascorbic acid, pH 6.0), viral particles were concentrated using Amicon Ultra $100 \mathrm{kDa}$ centrifugal devices (Millipore), treated with DNase I $(100 \mathrm{U} / \mathrm{mL})$ followed by the addition of $0.1 \mathrm{M}$ EDTA and $0.1 \mathrm{M}$ EGTA to halt enzyme activity, and extracted as previously described ${ }^{48}$. Briefly, viral particle suspensions were treated with Wizard PCR Preps DNA Purification Resin (Promega, WI, USA) at a ratio of $0.5 \mathrm{~mL}$ sample to $1 \mathrm{~mL}$

295 resin, and eluted with TE buffer (10 mM Tris, pH 7.5, $1 \mathrm{mM}$ EDTA) using Wizard Minicolumns. Extracted DNA was Covaris-sheared and size selected to 160-180 bp, followed by amplification and ligation per the standard Illumina protocol. Sequencing was done on a HiSeq 2000 system (101 bp, paired end reads) at the Genoscope facilities (Paris, France).

Temperature, salinity, and oxygen data were collected from each station using a CTD (Sea-Bird

300 Electronics, Bellevue, WA, USA; SBE 911plus with Searam recorder) and dissolved oxygen sensor (Sea-Bird Electronics; SBE 43). Nutrient concentrations were determined using segmented flow analysis $^{49}$ and included nitrite, phosphate, nitrite plus nitrate, and silica. Nutrient concentrations below the detection limit $\left(0.02 \mu \mathrm{mol} \mathrm{kg}{ }^{-1}\right)$ are reported as $0.02 \mu \mathrm{mol} \mathrm{kg} \mathrm{k}^{-1}$. All data from the Tara Oceans expedition are available from ENA (for nucleotide) and from PANGAEA (for environmental, biogeochemical, taxonomic and morphological data $)^{50-52}$.

\section{Malaspina expedition}

Thirteen bathypelagic samples and one mesopelagic sample were collected between April 19, 2011 and July 11, 2011 during the Malaspina 2010 global circumnavigation covering the Pacific and the 310 North Atlantic Ocean. All samples were taken at 4,000 m depth except two samples from stations 81 and 82 collected at 3,500 and 2,150 m respectively (Supplementary Table 1). Additionally, Station M114 was sampled at the OMZ region at $294 \mathrm{~m}$ depth. For each sample, $80 \mathrm{~L}$ of seawater were 0.22 $\mu \mathrm{m}$-filtered and viruses were concentrated from the filtrate using iron chloride flocculation ${ }^{47}$ followed by storage at $4^{\circ} \mathrm{C}$. More details about the sampling and additional variables used in the Malaspina expedition can be found $\mathrm{in}^{53}$. Further processing was done as for the Tara Oceans samples, except that Illumina sequencing was done at DOE JGI Institute (151 bp, paired end reads).

\section{Dataset generation}

\section{Contigs assembly}

320 An overview of the contigs generation process is provided in Supplementary Fig. 7. The first step consisted in the generation of a set of contigs using as many reads as possible from 104 oceanic viromes, including 74 epipelagic and 16 mesopelagic samples from the Tara Oceans expedition ${ }^{6}$, and 1 mesopelagic and 13 bathypelagic from the Malaspina expedition ${ }^{7}$. This set of contigs was generated through an iterative cross-assembly ${ }^{15}$ (using MOCAT $^{54}$ and Idba_ud ${ }^{55}$, Supplementary Fig. 7) as follows: (i) high-quality (HQ) reads were first assembled sample by sample with the MOCAT pipeline 
as described $\mathrm{in}^{21}$, (ii) all reads not mapping (Bowtie $2^{56}$, options --sensitive, -X 2000, and --nondeterministic, other parameters at default) to a MOCAT contig (by which we denote 'scaftigs', that is, contigs that were extended and linked using the paired-end information of sequencing read $^{57}$ ) were assembled sample by sample with Idba_ud (iterative k-mer assembly, with k-mer increasing from 20 to

330100 by step of 20), (iii) all reads remaining unmapped to any contig were then pooled by Longhurst province (i.e. unmapped reads from samples corresponding to the same Longhurst province were gathered) and assembled with Idba_ud (with the same parameters as above), and (iv) all remaining reads unmapped from every samples were gathered for a final cross-assembly (using Idba_ud). This resulted in 10,845,515 contigs (Supplementary Fig. 7B).

\section{Genome binning and re-assembly}

The contigs assembled from the marine viral metagenomes might still contain redundant sequences derived from the same, or closely related populations. We set out to merge contigs derived from the same population into clusters representing population genomes. To this end, contig sequences were first clustered at 95\% global average nucleotide identity (ANI) with cd-hit-est ${ }^{58}$ (options -c 0.95 -G 1 -n 10 -mask NX, Supplementary Fig. 7B), resulting in 10,578,271 non-redundant genome fragments. Next, we used co-abundance and nucleotide usage profiles of the non-redundant contigs to further identify contigs derived from the same populations with Metabat ${ }^{59}$. Briefly, Metabat uses Pearson correlation between coverage profiles (determined from the mapping of HQ reads of each sample to the contigs with Bowtie $2^{56}$, options --sensitive, -X 2000, and --non-deterministic, other parameters at default) and tetranucleotide frequencies (Metabat parameters: 98\% minimum correlation, mode "sensitive", see Supplementary Text for more detail about the selection of these parameters) to identify contigs originating from the same genome. The 8,744 bins generated, including 3,376,683 contigs, were further analyzed, alongside 623,665 contigs not included in any genome bin but $\geq 1.5 \mathrm{~kb}$.

350 In an attempt to better assemble these genome bins, two additional sets of contigs were generated for each genome bin, two additional sets of contigs were generated (beyond the set of initial contigs binned by Metabat ${ }^{59}$ ), based on the de novo assembly of (i) all reads mapping to the contigs in the genome bin, and (ii) only reads from the sample displaying the highest coverage for the genome bin (both assemblies with Idba_ud ${ }^{55}$, Supplementary Fig. 7C). The latter might be expected to lead to the "cleanest" genome assembly because it includes the minimum between-sample sequence variation, lowering the probability of generating chimeric contig ${ }^{60}$. The former may be necessary if the virus is locally rare, so that sequences from multiple metagenomes are needed to achieve complete genome coverage. Thus, if the assembly from the single "highest coverage sample" was improved or equivalent (longest contig in the new assembly representing $\geq 95 \%$ of the longest contig in the initial assembly), 360 this set of contigs was selected as the sequence for this bin $(n=6,423)$. This optimal single-sample assembly was thus privileged compared to a cross-assembly (either based on the initial contigs or on the re-assembly of all sequences aligned to that bin). Otherwise, the "all samples" bin re-assembly was selected if equivalent or better than the initial assembly (longest contig representing $\geq 95 \%$ of the longest initial contig, $\mathrm{n}=999$ ). The assumption that cross-assembly would be needed for locally rare

365 viruses, without a high-coverage sample, was confirmed by the comparison between the highest coverage of these two types of bins: on average, bins for which the "optimal" assembly were selected displayed a maximum coverage of 5.47 per Gb of metagenome, while the bins for which the "crossassembly" was selected displayed a maximum coverage of 1.37 per Gb of metagenome (Supplementary Table 2). Finally, if both re-assemblies yielded a longest contig smaller $(<95 \%)$ than the one in the 370 initial assembly, the bin was considered as a false positive (i.e. binning of contigs from multiple genomes, $n=1,356)$, and contigs from the initial assembly were considered as "unbinned" (263,006 contigs, added to the 623,665 contigs $\geq 1.5 \mathrm{~kb}$ retained as "unbinned"). 
375 VirSorter ${ }^{61}$ was used to identify microbial contigs (using the "virome decontamination" mode, with every contig $\geq 10 \mathrm{~kb}$ and not identified as a viral contig being considered as a microbial contig). Sequences with a prophage predicted were manually curated to distinguish actual prophages (i.e. viral regions within a microbial contig) from contigs that belonged to a viral genome and were wrongly predicted as a prophage. Contigs originating from an eukaryotic virus were identified based on best BLAST hit affiliation of the contig predicted genes against NCBI RefseqVirus (see Supplementary Text).

The genome bins were affiliated as microbial (if 1 or more contigs were identified as microbial, $\mathrm{n}=1,763$ ), eukaryotic virus (if contigs affiliated as eukaryotic virus comprised more than $10 \mathrm{~kb}$ or more than $25 \%$ of the genome bin total length, $n=962$ ) or viral (i.e. archaeal and bacterial viruses, $n=4,341$ ), with the 356 remaining bins, lacking a contig long enough for an accurate affiliation, considered as "unknown".

Viral bins were then refined to evaluate if they corresponded to a single or a mix of viral population(s). To that end, the Pearson correlation and Euclidean distance between abundance profiles (i.e. profile of the contig average coverage depth across the 104 samples) of bin members and the bin seed (i.e. the largest contig) were computed, and a single-copy viral marker gene (TerL) was identified in binned contigs (Supplementary Fig. 7E). Thresholds were chosen to maximize the number of bins with exactly one TerL gene and minimize the number of bins with multiple TerL genes (Supplementary Fig. 7G). For each bin, contigs with a Pearson correlation coefficient to the bin seed $<0.96$ or a Euclidean distance to the seed $>1.05$ were removed from the bin, and added to the pool of unbinned 395 contigs. Eventually, every bin still displaying multiple TerL genes after this refinement step were split, and all corresponding contigs added to the pool of "unbinned" contigs (Supplementary Fig. 7E).

The final set of contigs was formed by compiling (i) all contigs belonging to a viral bin, (ii) "unbinned" viral contigs (i.e. contigs affiliated to archaeal and bacterial virus and not part of any genome bin), and (iii) viral contigs identified in microbial or eukaryote virus bins (considered as 400 "unbinned" contigs, Supplementary Fig. 7F). Within this set of contigs, all viral bins were considered as viral populations, as well as every unbinned viral contig $\geq 10 \mathrm{~kb}$, leading to a total of 15,222 epi- and mesopelagic populations, and 58 bathypelagic populations (Supplementary Fig. 1, Supplementary Table 2, and Supplementary Text). In this study, we focus only on the 15,222 epi- and mesopelagic populations, totaling 24,353 contigs. For the detection of AMGs, we added to these populations all short epi- and mesopelagic unbinned viral contigs ( $<10 \mathrm{~kb})$, adding up to a total of 298,383 contigs.

\section{Sequence clustering and annotations}

Dataset of publicly available viral genomes and genome fragments

Genomes of viruses associated with a bacterial or archaeal host were downloaded from NCBI

410 RefSeq (1,680 sequences, v70, 05-26-2015). To complete this dataset of reference genomes, viral genomes and genome fragments available in Genbank, but not yet in Refseq were downloaded (July 2015) and manually curated to select only bacterial and archaeal viruses (1,017 sequences). These included viral genomes not yet added to RefSeq, as well as genome fragments from fosmid libraries generated from seawater samples ${ }^{12,13}$. Mycophage sequences (available from http://phagesdb.org ${ }^{62}$ ) and

415 not already in RefSeq were downloaded (July 2015) and included as well (734 sequences). Finally, 12,498 viral genome fragments from the VirSorter Curated Dataset, identified in publicly available microbial genome sequencing projects, were added to the database ${ }^{9}$.

\section{Genome (fragments) clustering through gene-content based network analysis}

$420 \quad$ Proteins predicted from 14,650 large GOV contigs ( $\geq 10 \mathrm{~kb}$ and $\geq 10$ genes), were added to all proteins from the publicly available viral genomes and genomes fragments gathered, and compared through all-vs-all blastp, with a threshold of $10^{-5}$ on e-value and 50 on bit score. Protein clusters were then defined using MCL (using default parameters for clustering of proteins, similarity scores as 
log-transformed e-value, and MCL inflation of $2^{63}$ ). vContact (https://bitbucket.org/MAVERICLab/ vcontact) was then used to calculate a similarity score between every pair of genome and/or contigs based on the number shared of PCs between the two sequences (as in ${ }^{8,9}$ ), and then compute a MCL clustering of the genomes/contigs based on these similarity scores (thresholds of 1 on similarity score, MCL inflation of 2). The resulting viral clusters (or VCs, clusters including $\geq 2$ contigs and/or genomes), consistent with a clustering based on whole-genome BLAST comparison, corresponded to

430 approximately genus-level taxonomy, with rare cases closer to subfamily-level taxonomy (Extended Data Fig. 2 and Supplementary Text). A total of 1,259 viral clusters were obtained, with 867 including at least one GOV sequence.

\section{Viral contigs annotation}

435 A functional annotation of all GOV predicted proteins was based on a comparison to the PFAM domain database (v27 $7^{64}$ ) with $\mathrm{HmmSearch}^{65}$ (threshold of 30 on bit score and 1e-3 on e-value), and additional putative structural proteins were identified through a BLAST comparison to protein clusters detected in viral metaproteomics dataset ${ }^{66}$. This metaproteomics dataset led to the annotation of 13,547 hypothetical proteins lacking a PFAM annotation. A taxonomic annotation was performed based on a

440 blastp of the predicted proteins against proteins from archaeal and bacterial viruses from NCBI RefSeq and Genbank (threshold of 50 on bit score and $10^{-3}$ on e-value).

VCs were affiliated based on isolate genome members, when available. When multiple isolates were included in the $\mathrm{VC}$, the $\mathrm{VC}$ was affiliated to the corresponding subfamily or genus of these isolates (excluding all "unclassified" cases). This was the case for VC_2 (T4 subfamily ${ }^{17,18}$ ), and VC_9

$445\left(\right.$ T7virus $\left.^{19}\right)$. When only one or a handful of affiliated isolate genomes were included in the VC and lacked genus-level classification, a candidate name was derived from the isolate (if several isolates, from the first one isolated). This was the case for VC_5 ( Cbaphi381virus $\left.^{67}\right), \mathrm{VC} 12\left(\right.$ P12024virus $\left.^{68}\right)$, VC_14 (MED4-117virus), VC_19 (HMO-2011virus $\left.{ }^{69}\right)$, VC_31 (RM378virus $\left.{ }^{70}\right)$, VC_36 $\left._{\text {(GBK2virus }}{ }^{71}\right)$, VC_47 (Cbaphi142virus $\left.{ }^{67}\right)$, and VC_277 (vB_RglS_P106Bvirus $\left.{ }^{72}\right)$. Otherwise, VCs were considered as "new VCs".

\section{"Phage proteomic tree" (i.e. "whole-genome comparison tree”) computation and visualization}

All publicly available complete genomes (see above), all complete (circular) and near-complete (extrachromosomal genome fragment $>50 \mathrm{~kb}$ with a terminase) from the VirSorter Curated Dataset, and all complete and near-complete GOV contigs were compared to generate a phage proteomic tree, as previously described ${ }^{12,73}$. Briefly, a proteomic similarity score was calculated for each pair of genome based on a all-vs-all tblastx similarity as the sum of bit scores of significant hits between two genomes (e-value $\leq 0.001$, bit score $\geq 30$, identity percentage $\geq 30$ ). To normalize for different genome sizes, each genome was also compared to itself to generate a self-score, and the distance between two 460 different genomes was calculated as a Dice coefficient (as in ${ }^{12}$ ), i.e. for two genomes A and B with a proteomic similarity score of $A B$, the corresponding distance $d$ would be $1-(2 * A B) /(A A+B B)$, with $A A$ and $\mathrm{BB}$ being the self-score of genomes $\mathrm{A}$ and $\mathrm{B}$ respectively. For clarity, the tree displayed in Extended Data Fig. 2 only include non-GOV sequences found in a VC with GOV sequence(s) or within a distance $<0.5$ to a GOV sequence, adding for a total of 1,522 reference sequences. iTOL ${ }^{74,75}$ was used 465 to visualize and display the tree.

\section{Distribution and relative abundance of viral populations and VCs \\ Detection and estimation of abundance for viral contigs and populations}

The presence and relative abundance of a viral contig in a sample were determined based on the 470 mapping of HQ reads to the contig sequences, computed with Bowtie 2 (options --sensitive, -X 2000, and --non-deterministic, default parameters otherwise ${ }^{56}$ ), as previously described ${ }^{10}$. A contig was considered as detected in a metagenome if more than $75 \%$ of its length was covered by aligned reads 
derived from the corresponding sample. A normalized coverage for the contig was then computed as the average contig coverage (i.e. number of nucleotides mapped to the contig divided by the contig length) normalized by the total number of bp sequenced in this sample.

The detection and relative abundance of a viral population was based on the coverage of its contigs: a population was considered as detected in a sample if more than $75 \%$ of its cumulated length was covered, and its normalized coverage was computed as the average normalized coverage of its contigs.

480

\section{Relative abundance of $V C s$}

The relative abundance of VCs was calculated based on the coverage of its members within the 15,222 viral populations identified. If a population included contigs all linked to the same VC, or linked to a single VC except for unclustered (because too short) contigs, this population coverage was 485 added to the total of the corresponding VC. In the rare cases where the link between population and VC was ambiguous because different contigs within a population pointed toward different VCs $(n=475$, i.e. $3.1 \%$ of the populations), the population coverage was equally split between these VCs. Finally, if no contig in the population belonged to any $\mathrm{VC}(\mathrm{n}=2,605,17 \%$ of the populations), the population coverage was added to the "unclustered" category. Eventually, for each sample, the cumulated coverage

490 of a VC was normalized by the total coverage of all populations to calculate a relative abundance of the $\mathrm{VC}$ among viral populations.

The selection of abundant VCs within a sample was based on the contribution of the VC to the sample diversity as measured by the Simpson index. For each sample, the overall Simpson index was first calculated with all VCs. Then, VCs were sorted by decreasing relative abundance and 495 progressively added to a new calculation of the Simpson index. VCs considered as abundant were the ones which, once cumulated, represented $80 \%$ of the sample diversity (i.e. a Simpson index greater or equal to $80 \%$ of the sample total Simpson index, Extended Data Fig. 1C). The 38 VCs identified as abundant in at least 2 different stations were selected as "recurrently abundant VCs in the GOV dataset" (Fig. 2 and Extended Data Fig. 3).

\section{Host prediction and diversity}

A genome database of putative hosts for the epi- and mesopelagic GOV viruses was generated, including all archaea and bacteria genomes annotated as "marine" from NCBI RefSeq and WGS (both times only sequences $\geq 5 \mathrm{~kb}, 184,663$ sequences from 4,452 genomes, downloaded in August 2015), and

505 all contigs $\geq 5 \mathrm{~kb}$ from the 139 Tara Oceans microbial metagenomes corresponding to the bacteria and archaea size fraction (791,373 sequences $)^{21}$. For these microbial metagenomic contigs, a first blastn was computed to compare them to all GOV contigs, and exclude from the putative host dataset all metagenomic contigs with a significant similarity to a viral GOV sequence (thresholds of 50 on bit score, 0.001 on e-value, and $70 \%$ on identity percentage) on $\geq 90 \%$ of their length, as these are likely

510 sequences of viral origin sequenced in the bacteria and archaea size fraction (these represented $2.2 \%$ of the contigs in the assembled microbial metagenomes). The taxonomic affiliation of NCBI genomes was taken from the NCBI taxonomy. For Tara Oceans contigs, a last common ancestor (LCA) affiliation was generated for each contig based on genes affiliation ${ }^{21}$, if 3 genes or more on the contig were affiliated. Three different approaches were then used to link viral contigs and putative host genomes

515 (see Supplementary Text and ref. 76 for an extended discussion about the efficiency and raw results of these host prediction methods, and Supplementary Table 4 for a list of all host predictions by sequence).

\section{BLAST-based identification of sequence similarity between viral contigs and host genome}

520 All GOV viral contigs were compared to all archaeal and bacterial genomes and genome fragments with a blastn (threshold of 50 on bit score and 0.001 on e-value), to identify regions of similarity 
between a viral contig and a microbial genome, indicative of a prophage integration or horizontal gene transfer ${ }^{76}$. A host prediction was made when (i) a NCBI genomes displayed a region similar to a GOV viral contig $\geq 5 \mathrm{~kb}$ at $\geq 70 \%$ id, or (ii) when a Tara Oceans microbial metagenomic contig ( $\geq 5 \mathrm{~kb})$ displayed a region similar to a GOV viral contig $\geq 2.5 \mathrm{~kb}$ at $\geq 70 \%$ id.

\section{Matches between GOV viral contigs and CRISPR spacers.}

CRISPR arrays were predicted for all putative host genomes and genome fragments (NCBI microbial genomes and Tara Oceans microbial metagenomic contigs) with MetaCRT ${ }^{77,78}$. CRISPR 530 spacers were extracted, and all spacers with ambiguous bases or low complexity (i.e. consisting of 4 to 6 bp repeat motifs) were removed. All remaining spacers were matched to viral contigs with fuzznuc ${ }^{79}$, with no mismatches allowed, which although rarely observed yields highly accurate host predictions ${ }^{76}$.

\section{Nucleotide composition similarity: comparison of tetranucleotide frequency}

535 Bacterial and archaeal viruses tend to have a genome composition close to the genome composition of their host, a signal that can be used to predict viral-host pairs ${ }^{9,76}$. Here, canonical tetranucleotide frequencies were observed for all viral and host sequences using Jellyfish ${ }^{80}$, and mean absolute error (i.e. average of absolute differences) between tetranucleotide frequency vectors were computed with in-house Perl and Python scripts for each pair of viral and host sequence. A GOV viral contig was then 540 assigned to the closest sequence (i.e. lowest distance d) from the pool of NCBI genomes if $d<0.001$ (because both the tetranucleotide frequency signal and the taxonomic affiliation of these complete genomes are more robust than for metagenomic contigs), and otherwise assigned to the closest (i.e. lowest distance) Tara Oceans microbial contig if $\mathrm{d}<0.001$.

545 Summarizing host prediction at the VC level

Overall, 3,675 GOV contigs could be linked to a putative host group among the 24,353 GOV contigs associated with an epi- or mesopelagic viral population. To summarize these affiliations at the VC level, a Poisson distribution was used to estimate the number of expected false positive associations for each VC - host group combination based on (i) the global probability of obtaining a host prediction across all pairs of viral and host sequences tested and for all methods $\left(p=5.8 \times 10^{-08}\right)$, (ii) the number of potential predictions generated for the VC, corresponding to 3 times the number of sequences in the VC (to take into account the three methods), and (iii) the number of sequences from the host group in the database. By comparing the number of links observed between a VC and a host group to this expected value, which takes into account the bias in database (i.e. some host groups will be over- or underrepresented in our set of archaeal and bacterial genomes and genome fragments) and the bias linked to the variable number of sequences in VCs, we can determine if the number of associations observed for any $\mathrm{VC}$ - host group combination is likely to be due to chance alone (and calculate the associated pvalue).

\section{Microbial community diversity and richness indexes}

Diversity and richness indexes for putative host populations were based on the OTU abundance matrix generated from the analysis of ${ }_{m i}$ TAGs in Tara Oceans microbial metagenomes ${ }^{21}$. These indexes were computed for each host group at the same taxonomic level as the host prediction, i.e. the phylum level except for Proteobacteria where the class level is used. The $\mathrm{R}$ package vegan ${ }^{81}$ was used to estimate for each group (i) a global Chao index (i.e. including all OTUs from all samples) through the function estaccumR, (ii) a sample-by-sample Chao index with the function estimateR, and (iii) Sorensen indexes between all pairs of samples with the function betadiver. Diversity indexes presented in Extended Data Fig 4 were based on epipelagic samples only, as the 38 VCs identified as abundant were mostly retrieved in epipelagic samples. Candidate division OP1 was excluded from this analysis because no OTU affiliated to this phylum was identified. 


\section{Identification and annotation of putative AMGs}

Detection of $A M G s$

Predicted proteins from all GOV viral contigs were compared to the PFAM domain database (hmmsearch ${ }^{65}$, threshold of 40 on bit score and 0.001 on e-value), and all PFAM domains detected were classified into 8 categories: "structural", "DNA replication, recombination, repair, nucleotide metabolism", "transcription, translation, protein synthesis", "lysis", "membrane transport, membraneassociated", "metabolism", "other", and "unknown" (as in ${ }^{24}$ ). Four AMGs (i.e. similar to a domain from the "metabolism" category) were then selected for further study because of their central role in sulfur ( $d s r \mathrm{C}$ and sox $\mathrm{YZ}$ ) or nitrogen (P-II, amoC) cycle, and the fact that these had never been detected in a surface ocean viral genome so far ( $d s r C / t u s E$-like genes have been detected in deep water viruses $^{14,29}$ ). To evaluate if an AMG was "known", a list of PFAM domain detected in NCBI RefSeqVirus and Environmental Phages was computed based on a similar hmmsearch comparison (threshold of 40 on bit score and 0.001 on e-value), and augmented by manual annotation of AMGs from $^{24,82}$. The complete list of PFAM domains detected in GOV viral contigs is available as Supplementary Table 5.

\section{Phylogenetic tree generation and contigs map comparison}

Sequences similar to these AMGs were recruited from the Tara Oceans microbial metagenomes ${ }^{21}$ based on a blastp of all predicted proteins from microbial metagenome to the viral AMGs identified (threshold of 100 on bit score, $10^{-5}$ on e-value, except for P-II where a threshold of 170 on bit score was used because of the high number of sequences recruited). The viral AMG sequences were also compared to NCBI nr database (blastp, threshold of 50 on bit score and $10^{-3}$ on e-value) to recruit relevant reference sequences (up to 20 for each viral AMG sequence). These sets of viral AMGs and related protein sequences were then aligned with Muscle ${ }^{83}$, the alignment manually curated to remove poorly aligned positions with Jalview ${ }^{84}$, and two trees were computed from the same curated alignment: a maximum-likelihood tree with FastTree (v2.7.1, model WAG, other parameters set to default ${ }^{85}$ ) and a bayesian tree with MrBayes (v3.2.5, mixed evolution models, other parameters set to default, 2 MCMC chains were run until the average standard deviation of split frequencies was $<0.015$, relative burn-in of $25 \%$ used to generate the consensus tree ${ }^{86}$ ). In all cases except AmoC, the mixed model used by MrBayes was $100 \%$ WAG, confirming that this model was well suited for archaeal and bacterial virus protein trees. Manual inspection revealed only minor differences between each pair of trees, so an SH test was used to determine which tree best fitted the sequence alignment, using the $\mathrm{R}$ library phangorn $^{87}$. Itol ${ }^{74}$ was used to visualize and display these trees, in which branches with supports $<40 \%$

605 were collapsed. Annotated interactive trees are available online at http://itol.embl.de/shared/Siroux. Contigs map comparison were generated with Easyfig ${ }^{88}$, following the same method as for the VCs (see Supplementary Information).

\section{Functional characterization of putative AMGs}

610 Conserved motifs were identified on the different AMGs based on the literature: $d s r \mathrm{C}$ conserved motifs were obtained from ref. 33, soxYZ conserved residues were identified from the PFAM domains PF13501 and PF08770, and P-II conserved motifs from PROSITE documentation PDOC00439. A 3D structure could also be predicted for P-II AMGs by I-TASSER ${ }^{89}$ (default parameters), the quality of these predictions being confirmed with ProSA web server ${ }^{90}$. To further confirm the functionality of

615 these genes, selective constraint on these AMGs was evaluated through $\mathrm{pN} / \mathrm{pS}$ calculation, as in ref. 91. Briefly, synonymous and non-synonymous SNPs were observed in each AMG, and compared to expected ratio of synonymous and non-synonymous SNPs under a neutral evolution model for this genes. The interpretation of $\mathrm{pN} / \mathrm{pS}$ is similar as for $\mathrm{dN} / \mathrm{dS}$ analyses, with the operation of purifying selection leading to $\mathrm{pN} / \mathrm{pS}$ values $<1$. Finally, AMG transcripts were searched in metatranscriptomic 
620 datasets generated through the Tara Oceans consortium (ENA Id ERS1092158, ERS488920, and

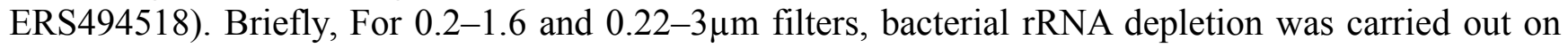
240-500 ng total RNA using Ribo-Zero Magnetic Kit for Bacteria (Epicentre, Madison, WI). The Ribo-Zero depletion protocol was modified to be adapted to low RNA input amounts ${ }^{92}$. Depleted RNA was used to synthetize cDNA with SMARTer Stranded RNA-Seq Kit (Clontech, Mountain View,

$625 \mathrm{CA})^{92}$. Metatranscriptomic libraries were quantified by qPCR using the KAPA Library Quantification Kit for Illumina Libraries (KapaBiosystems, Wilmington, MA) and library profiles were assessed using the DNA High Sensitivity LabChip kit on an Agilent Bioanalyzer (Agilent Technologies, Santa Clara, CA). Libraries were sequenced on Illumina HiSeq2000 instrument (Illumina, San Diego,CA) using 100 base-length read chemistry in a paired-end mode. High quality reads were then mapped to viral contigs

630 containing $d s r C$, soxYZ, P-II, or amoC genes with SOAPdenovo $2^{57}$ within MOCAT ${ }^{54}$ (options screen and filter with length and identity cutoffs of 45 and 95\%, respectively, and paired-end filtering set to yes), and coverage was defined for each gene as the number of bp mapped divided by gene length (including only reads mapped to the predicted coding strand).

\section{Distribution of AMGs and association with geochemical metadata}

The distribution and relative abundance of AMGs was based on the read mapping and normalized coverage of the contig including the AMG. To get a range of temperature and nutrient concentrations for the widespread AMGs (detected in $>5$ stations) that takes into account both the samples in which these AMGs were detected and the differences in normalized coverage, a set of samples was selected

640 through a weighted random drawing replacement, with the weight of each sample corresponding to the AMG's normalized coverage. That way, a range of temperature or nutrient concentration values associated with the AMG's distribution and abundance could be generated for each AMG and each environmental parameter tested. The number of samples randomly selected for each AMG was the same as the total number of samples for which a value of this parameter was available.

\section{Scripts and data availability}

Scripts used in this manuscript are available on the Sullivan lab bitbucket under project "GOV_Ecogenomics" (https://bitbucket.org/MAVERICLab/gov_ecogenomics). All raw reads are available through ENA (Tara Oceans) or JGI (Malaspina) using the dataset Ids listed in Supplementary

650 Table 1. Processed data are available through iVirus, including all sequences from assembled contigs, list of viral populations and associated annotated sequences as genbank files, viral clusters composition and characteristics, map comparisons of genomes and contigs of the 38 abundant VCs, and host predictions for viral contigs.

\section{Extended Data}

Extended Data Figure 1: Accumulation curves of populations (A) and viral clusters (VCs, B) and identification of abundant VCs in GOV samples (C). A \& B. Accumulation curves were computed from 50 random shuffling of samples (blue dots), either with all, epipelagic, mesopelagic, or 660 bathypelagic samples. For each curve, the average of 50 iterations is highlighted with red dots. C. Schematic of the selection process of abundant VCs. For each sample, VCs accounting for (up to) $80 \%$ of the sample diversity (as assessed by Simpson index) were considered as abundant (example for sample 125_MIX on the left). VCs detected as abundant in at least two different stations were included in the $38 \mathrm{VC}$ s described in Fig. 2 and Extended Data Fig. 3.

Extended Data Figure 2: Comparison of VCs with other classification methods: phage proteomic tree and percentage of shared genes. The phage proteomic tree includes the $756 \mathrm{GOV}$ complete and near-complete genomes from epi- and mesopelagic samples, and closest references from RefSeq and 
Environmental phages $(\mathrm{d}<0.5$ to a GOV sequence or found in the same VC as a GOV sequence). All VCs with more than 8 representatives in the tree or part of the 38 abundant VCs are indicated with coloring of the outer ring. The name and affiliation (if available) of the 38 abundant VCs are indicated next to the $\mathrm{VC}$ on the colored ring. Branches of monophyletic clades including more than $3 \mathrm{GOV}$ and/or uncultivated marine sequences with no isolate reference are highlighted in blue. Inset: distribution of number of shared genes (i.e. number of shared protein clusters) for viral genome/contigs

675 pairs either between different VCs or within VCs.

Extended Data Figure 3: Summary of 34 of the 38 abundant viral clusters (VCs, the 4 other abundant VCs being the ubiquitous ones presented in Fig. 2). Predicted genome size is based on the set of isolates and circular contigs in the VC (NA corresponds to VCs without any circular contigs, or

680 for which the relative standard deviation of estimated genome size across the different isolate(s) and/or circular contigs is greater than 15\%). Host association values are based on the number of cluster members associated with each host group, the statistical significance of this number of predictions being evaluated by comparison with an expected number of associations calculated from a Poisson distribution. Host associations based on known isolates are indicated with a star (for associations based

685 on cultivated isolates) or a dot (for associations based on the detection of a cluster member in a microbial genome from the VirSorter Curated Dataset). The abundant epipelagic microbial groups (representing $>1 \%$ of the microbial OTUs abundance of epipelagic samples) are highlighted in bold. Distribution and relative abundance of VCs are based on the cumulated coverage of VC members among sample viral populations. The main oceanic basins are indicated for each set of sample, Med.

690 Sea-Mediterranean Sea.

Extended Data Figure 4: Association between abundant viral clusters (VCs) and host group abundance and diversity $\mathbf{A}$. Abundance and diversity of bacterial and archaeal host groups associated with the 38 abundant VCs (see Fig. 2A). For each host group (phylum level, except for Proteobacteria

695 where the class level is used), the different panels display from top to bottom (i) the number of VCs associated with this host group, (ii) the global relative abundance of this group estimated from the microbial metagenomic OTU counts, (iii) the global diversity of this group based on a Chao index computation including all Tara Oceans microbial metagenome samples (i.e. including both Alpha and Beta diversity), (iv) the distribution of Chao indexes by sample for this group (Alpha diversity), and (v)

700 the average Sorensen index between pairs of samples including at least one OTU of this group (Beta diversity). OTU counts were derived from the 109 epipelagic microbial metagenomes described $\mathrm{in}^{21}$. B. Pearson correlations between host group relative abundance or diversity indexes (Global Chao, Average Chao across samples, and Average Sorensen across samples) and the number of VCs.

705 Extended Data Figure 5: Diversity, distribution, and genome context of $d s r C$ genes in GOV contigs. A. Maximum-likelihood tree (from an amino-acid alignment) including the 11 viral DsrC and microbial sequences from microbial metagenomes and NCBI $\mathrm{nr}$ database. The presence of conserved C residues (named Cys-A \& Cys-B, as $\mathrm{in}^{33}$ ) is indicated with color circles next to each sequence or clade, and the corresponding type of DsrC-like protein is indicated by coloring the branch or clade. The 710 microbial metagenomic contigs affiliated to uncultivated, marine sulfur-oxidizing Gammaproteobacteria (as confirmed by complementary phylogenetic analysis of DsrAB, Supplementary Fig. 6) are indicated with a star next to the sequence or clade. Viral AMG sequences are highlighted in blue, internal nodes SH-like supports are represented by proportional circles (all nodes with support $<0.40$ were collapsed). Each $d s r C$ AMG is associated with an abundance profile (on the 715 right) displaying the relative abundance of the contig across the 91 epi- and mesopelagic samples (based on normalized coverage, i.e. contig coverage / Gb of metagenome). B. Comparison of $d s r C$ containing contigs maps. T4-like marker genes (PhoH and T4 baseplate) are indicated on the maps, 
alongside putative AMGs (Fe-S biosyn for Iron-sulfur cluster biosynthesis, and Amt for Ammonia transporter).

Extended Data Figure 6: Diversity, distribution, and genome context of soxYZ genes in GOV contigs. A. Bayesian tree (from an amino-acid alignment) including the 4 viral SoxYZ and microbial sequences from microbial metagenomes and NCBI nr database. The affiliation of microbial clades (either from the NCBI reference or from the LCA affiliation of metagenomic contigs) is indicated by coloring of the grouped clades or with a colored square next to the sequence. Viral AMG sequences are highlighted in blue, posterior probabilities are represented by proportional circles (all nodes with posterior probability $<0.40$ were collapsed). Clades including sulfur-oxidation proteobacteria are indicated on the tree. Each soxYZ AMG is associated with an abundance profile (on the right) displaying the relative abundance of the contig across the 91 epi- and mesopelagic samples (based on

normalized coverage, i.e. contig coverage / Gb of metagenome). B. Comparison of soxYZ-containing contigs maps. For contig GOV_bin_4310_contig-100_0, the second largest contig from the same bin (GOV_bin_4310_contig-100_1) is displayed. T4-like marker genes (PhoH, Gp23 and T4 baseplate) are indicated on the maps, alongside putative AMGs (Fe-S biosyn: Iron-sulfur cluster biosynthesis).

735 Extended Data Figure 7: Diversity, distribution, and genome context of P-II genes in GOV contigs. A. Maximum-likelihood tree (from an amino-acid alignment) including the 10 viral P-II and microbial sequences from microbial metagenomes and NCBI $\mathrm{nr}$ database. The affiliation of microbial clades (either from the NCBI reference or from the LCA affiliation of metagenomic contigs) is indicated by coloring of the grouped clades or with a colored square next to the sequence. The 740 sequences lacking the conserved uridylation site of P-II (Supplementary Fig. 4) are highlighted with a star next to the sequence name or clade. Viral AMG sequences are highlighted in blue, internal nodes $\mathrm{SH}$-like supports are represented by proportional circles (all nodes with support $<0.40$ were collapsed). Each P-II AMG is associated with an abundance profile (on the right) displaying the relative abundance of the contig across the 91 epi- and mesopelagic samples (based on normalized coverage, i.e. contig

745 coverage / Gb of metagenome). B. Comparison of P-II-containing contigs maps. Ammonia transporter genes linked to P-II are indicated on the map (Amm Transp, dark red). When available, the VC affiliation of each contig is indicated next to the contig name. Contig GOV_bin_5834_contig-100_7 is too short to be clustered based on a shared PC network, however the seed contig of its population was clustered (in VC_12, Siphoviridae - P12024virus), hence this seed contig affiliation is indicated.

\section{0}

Extended Data Figure 8: Diversity, distribution, and genome context of amoC gene in GOV contigs. A. Maximum-likelihood tree (from an amino-acid alignment) including the GOV amoC AMG and microbial sequences from microbial metagenomes and NCBI $\mathrm{nr}$ database. The affiliation of microbial clades (either from the NCBI reference or from the LCA affiliation of metagenomic contigs)

755 is indicated by coloring of the grouped clades or with a colored square next to the sequence. Viral AMG sequence is highlighted in blue, internal nodes SH-like supports are represented by proportional circles (all nodes with support $<0.40$ were collapsed). B. Abundance profile displaying the relative abundance of the contig across the 91 epi- and mesopelagic samples (based on normalized coverage, i.e. contig coverage / Gb of metagenome). C. Map of the amoC-containing contig.

\section{0}

Extended Data Figure 9: Normalized coverage of contigs harboring AMG as function of the temperature and nutrient concentrations $\left(\mathrm{NO}_{2}, \mathrm{NO}_{3}, \mathrm{PO}_{4}\right)$ of the corresponding samples. AMGs are grouped by clade based on the phylogeny (see Extended Data Fig. 5-6-7), and coverages are cumulated when a clade included multiple contigs. Plots display the cumulated normalized coverage of 765 a clade (y-axis) as function of the temperature or nutrient concentration (x-axis) across all epipelagic samples (mesopelagic samples were excluded from the analysis since the AMG signal was detected in 
epipelagic samples), only for clades not geographically restricted (i.e. found in $>5$ samples, see Fig. 3C). Samples are color-coded according to their ocean and sea region (Supplementary Table 1). The calculated preferential range of temperature or nutrient concentration is displayed below each plot for the epipelagic AMGs (P-II-4 distribution could not be linked to specific environmental conditions, but this AMG is the only one consistently retrieved in mesopelagic samples).

Extended Data Table 1: Summary of genes and contigs characteristics for new viral DsrC, SoxYZ, and P-II AMGs. Each gene is linked to its contig, and when available, to the corresponding 775 viral population and predicted host (from BLAST hit, CRISPR spacer similarity, or nucleotide composition similarity). Widespread and abundant VCs are highlighted in bold. In addition, the calculated $\mathrm{pN} / \mathrm{pS}$ of each gene is indicated (measuring the strength of selection pressure occurring for this gene, the gene with a $\mathrm{pN} / \mathrm{pS}$ not representing a strong purifying selection is highlighted in red), as well as the coverage of these genes and other genes in the contigs in 3 metatranscriptomic samples

780 from 3 open ocean Tara stations (cases where the AMG coverage is $>0.5$ and associated with the coverage of other genes from the same viral contig are highlighted in green).

\section{Supplementary Information}

\section{Supplementary Text}

Supplementary Table 1: List of viromes included in the GOV dataset. For each virome, the corresponding expedition, station number, and depth is indicated. Tara Oceans stations are prefixed with "Tara_" and Malaspina stations with an "M". Accession numbers are given for raw reads available in ENA (for Tara Oceans samples) and on JGI IMG (for Malaspina samples). Longhurst provinces and biomes are defined based on Longhurst ${ }^{93}$ and environmental features are defined based on Environment Ontology (http://environmentontology.org/). The total number of reads and bp sequenced, as well as the number of bp mapped to viral contigs within and outside of populations are indicated. *Malaspina stations for which no water mass or basin data are available because these were not included in the previous study ${ }^{53}$.

Supplementary Table 2: GOV viral population summary. The number of contigs, total length and length of the largest contig, type of assembly used, and highest normalized coverage across the GOV samples is indicated in the first tab. For populations already identified in the TOV dataset (contigs similar at $95 \%$ ANI on $\geq 50 \%$ of their length), the size of the TOV contig is noted. In the second tab, the normalized coverage (average coverage of the population contig(s) normalized by the total sequencing depth of the sample) is indicated as coverage / Gb of metagenome for all GOV samples.

Supplementary Table 3: Summary of Viral Clusters (VCs). The first tab lists, for each VC, the 805 number of members (total, and by dataset, i.e. originating from RefSeq, environmental phages, VirSorter Curated Dataset, and GOV), alongside the affiliation of RefSeq members of the VCs (when available) at the family, subfamily, and genus levels. The second tab includes the cumulative normalized coverage of each VC in each sample (based on the coverage of populations members of the $\mathrm{VC}$ ), as well as the sum of coverage for the 38 recurrently abundant VCs and all other VCs at the bottom.

Supplementary Table 4: List of host prediction for GOV viral contigs associated with a population. For each prediction, the type of signal (blastn, CRISPR, tetranucleotide composition), the host sequence used for the prediction alongside its affiliation, and the strength of the prediction (length of the blastn match, number of mismatches in the CRISPR spacer, and distance between viral and host 
tetranucleotide frequencies vectors) is indicated.

Supplementary Table 5: List of PFAM domains detected in GOV viral contigs. For each PFAM domain, the number of genes detected in the GOV dataset is indicated, alongside the category of the 820 domain (as in ${ }^{24}$ ). The category "other" category includes PFAM domains with vague descriptions, multiple functions, or regulatory functions.

Supplementary Figure 1: Schematic of the different levels of organization used in this study. The base unit is the contig, i.e. assembled genome (fragment). These contigs are gathered (when available) 825 in viral populations, a proxy for viral "species", through genome binning based on co-occurrence and similarity in nucleotide composition. A higher level of organization (VCs, subfamily $\sim$ genus level) is achieved by clustering the contigs based on shared gene content.

Supplementary Figure 2: Multiple alignment of $\boldsymbol{d s r} \mathbf{C}$ protein sequences. Conserved residues are 830 indicated below the alignment, and the two conserved $\mathrm{C}$ residues representing the active sites of "true $d s r C "$ (Cys-B and Cys-A) are named as in ${ }^{33}$. Viral AMGs are highlighted in bold, with previously described anoxic SUP05 viruses sequences in red $\left(\right.$ from $\left.^{14,29}\right)$ and epipelagic GOV sequences in black.

Supplementary Figure 3: Multiple alignment of $\operatorname{sox} Y Z$ protein sequences. Conserved residues are 835 indicated below the alignment for SoxY and SoxZ protein domains, based on the respective PFAM domains (PF13501 and PF08770). Viral AMGs are highlighted in bold.

Supplementary Figure 4: Alignment (A) and predicted 3D structures (B) of P-II AMGs. Conserved motifs are indicated below the alignment (PROSITE: PDOC00439). The uridylation site is

840 highlighted with a star. Characterized structure (from E. Coli) and predicted 3D conformations are colored according to secondary structures (alpha helix: blue, beta strand: red), except for the trimer structure of E. Coli PII where each subunit is colored differently. For predicted structures, the model quality as assessed by ProSA ${ }^{90}$ is indicated below the model. Viral AMGs are highlighted in bold.

845 Supplementary Figure 5: Alignment (A) and predicted transmembrane domain (B) of amoC AMGs. The viral sequence is highlighted in bold, and conserved residues are indicated below the alignment. Transmembrane domains were predicted with $\mathrm{TMHMM}^{94}$ for the AMG amoC (left), and a reference amoC from the ammonia-oxidizing Nitrosopumilus maritimus SCM1 (right).

850 Supplementary Figure 6: Dissimilatory sulfite reductase $(\boldsymbol{d s} \boldsymbol{A} B)$ tree showing the phylogeny of

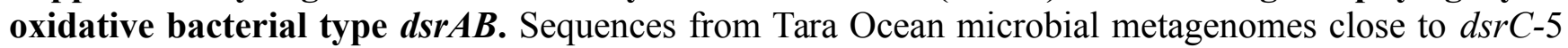
AMG are colored in blue and are affiliated with sulfur-oxidizing Gammaproteobacteria. Other phylogenetic groups and $d \operatorname{sr} A B$ families are collapsed and shown as triangles.

855 Supplementary Figure 7: Overview and result of the cross assembly, binning, and viral contigs selection process. A. Iterative assembly viromes. First, for each sample, reads were mapped to the set of contig generated through MOCAT ${ }^{54}$. Reads not assembled (i.e. not mapped to any contigs) were then used in another assembly, using Idba_ud ${ }^{55}$. Unmapped reads after this second round of sample-bysample assembly were then pooled by Longhurst province (i.e. all unmapped reads from all samples

860 within one province), and cross-assembled with Idba_ud ${ }^{15}$. Finally, all unmapped reads after this third round of assembly were gathered and assembled with Idba_ud. B. Results of the iterative assembly process. For each assembly round, the number of contigs is displayed alongside the cumulated percentage of reads mapped to a contig. C. Overview of the binning process. Contigs generated through the iterative assembly were binned based on correlation between their abundance profile and 
865 similarities between their tetranucleotide frequency (using Metabat ${ }^{59}$ ). For each bin, two contig pools (beyond the initial set of contigs) were generated, assembling either all reads mapping to the contig pool, or only reads from the sample in which the bin had the highest coverage (both assemblies computed with Idba_ud). The set of contigs including the largest genome fragment was then kept for each bin. D. Results of the re-assembly of bins. For each type of bin assembly (highest coverage

870 sample, all samples, or initial assembly) the number of bins for which this type was selected is indicated on top, with the distribution of increase in length of longest contig at the bottom. E. Bin refinement based on abundance profile similarities. For each bin, the abundance profile of each contig was compared to the abundance profile of the bin seed contig (largest contig), and contigs not well correlated to the bin seed were excluded. Bins still displaying multiple TerL gene (single-copy marker

875 gene for viruses) after this bin refinement step were split. F. Bin affiliation and viral population definition. Bins were either affiliated as entirely viral and considered as single viral populations, or included non-viral contigs, in which case viral contigs in these bins were considered as "unbinned" and selected as viral population seed if $\geq 10 \mathrm{~kb}$ G. Selection of thresholds for bin refinement based on abundance profile similarities. Thresholds to exclude contigs from bins based on Euclidean distance

880 and Pearson correlation coefficient between contig abundance profile and bin seed profile were explored, looking for the best compromise between number of true positive (z-axis, number of bins with a single TerL) and number of false negative (in colors, number of bins with multiple TerL). The thresholds combination chosen is indicated with a black square.

\section{Acknowledgements}

We thank Joshua Weitz for advice on statistics, Claus Pelikan for help with the DsrAB phylogenetic tree, Christiane Dahl for valuable discussion regarding DsrC function, and members of the Sullivan and Rich labs for suggestions and comments on this manuscript. We acknowledge support from UA highperformance computing and the Ohio Supercomputer Center. This viral research was funded by a 890 National Science Foundation grant (\#1536989) and Gordon and Betty Moore Foundation grants (\#3790, \#GBMF2631) to MBS. SR was partially supported by the University of Arizona Technology and Research Initiative Fund through the Water, Environmental and Energy Solutions Initiative and the Ecosystem Genomics Institute. BED was supported by the Netherlands Organization for Scientific Research (NWO) Vidi grant 864.14.004 and CAPES/BRASIL. AL was supported by the Austrian

895 Science Fund (FWF, project P25111-B22). We thank all people who prepared virus samples on board: Silvia G Acinas, Defne Arslan, Lucie Bittner, Julia Boras, Encarna Borruell, Christophe Boutte, Camille Clerissi, Montserrat Coll, Francisco M. Cornejo-Castillo, Cristina Díez Vives, Celine Dimier, Melissa Beth Duhaime, Isabel Ferrera, Laurence Garczarek, Nigel Grimsley, Pascal Hingamp, Lee Karp-Boss, Elena Lara, Noan LeBescot, Thomas Lefort, Hiroyuki Ogata, Eric Pelletier, Julie Poulain,

900 Frank Prejger, Guillem Salazar, Lucie Subirana, Anne Thompson, Dolors Vaqué, Emilie Villar, as well as Montse Sala for organizing the transfer and shipping of Malaspina samples. The Tara Oceans consortium effort was possible thanks to the commitment of the following people and sponsors: CNRS (in particular Groupement de Recherche GDR3280), European Molecular Biology Laboratory (EMBL), Genoscope/CEA, VIB, Stazione Zoologica Anton Dohrn, UNIMIB, Fund for Scientific

905 Research - Flanders, Rega Institute, KU Leuven, The French Ministry of Research, the French Government 'Investissements d'Avenir' programmes OCEANOMICS (ANR-11-BTBR-0008), FRANCE GENOMIQUE (ANR-10-INBS-09-08), MEMO LIFE (ANR-10-LABX-54), PSL* Research University (ANR-11-IDEX-0001-02), ANR (projects POSEIDON/ANR-09-BLAN-0348, PROMETHEUS/ANR-09-PCS-GENM-217, TARA-GIRUS/ANR-09-PCS-GENM-218), European

910 Union FP7 (MicroB3/No.287589), ERC Advanced Grant Award to CB (Diatomite: 294823), BIO5, and Biosphere 2 to MBS, Spanish Ministry of Science and Innovation grant CGL2011-26848/BOS and MicroOcean PANGENOMICS to SGA, TANIT (CONES 2010-0036) from the Agència de Gestió d 'Ajuts Universitaris i Recerca to SGA, JSPS KAKENHI Grant Number 26430184 to HO. We also 

Foundation, Region Bretagne, Lorient Agglomeration, World Courier, Illumina, the EDF Foundation, FRB, the Prince Albert II de Monaco Foundation, the Tara schooner and its captains and crew. We thank MERCATOR-CORIOLIS and ACRI-ST for providing daily satellite data during the expedition. We are also grateful to the French Ministry of Foreign Affairs for supporting the expedition and to the countries who graciously granted sampling permissions. Tara Oceans would not exist without 920 continuous support from 23 institutes (http://oceans.taraexpeditions.org). We also acknowledge excellent assistance from the European Bioinformatics Institute (EBI), as well as Yan Yuan and the EMBL IT core facility. Malaspina Expedition and Malaspinomics Projects are funded by the Spanish Ministry of Economy and Competitiveness (refs. CSD2008-00077 and CTM2011-15461-E) to CMD. Sequencing of the Malaspina viruses metagenomes was done at the Joint Genome Institute and was

925 supported by US Department of Energy Joint Genome Institute 2011 Microbes Program grant CSP 602 to SGA. The work conducted by the US Department of Energy Joint Genome Institute is supported by the Office of Science of the US Department of Energy under Contract No. DE-AC02-05CH11231. The authors further declare that all data reported herein are fully and freely available from the date of publication, with no restrictions, and that all of the samples, analyses, publications, and ownership of

930 data are free from legal entanglement or restriction of any sort by the various nations whose waters the Tara Oceans expedition sampled in. Data described herein is available at EBI and PANGAEA (see Supplementary Table 1). All authors approved the final manuscript. This article is contribution number XXX of the Tara Oceans Expedition.

\section{Tara Oceans Coordinators}

Silvia G. Acinas ${ }^{1}$, Peer Bork ${ }^{2,3}$, Emmanuel Boss ${ }^{4}$, Chris Bowler $^{5}$, Colomban de Vargas ${ }^{6,7}$, Michael Follows $^{8}$, Gabriel Gorsky ${ }^{9,31}$, Nigel Grimsley ${ }^{10,11}$, Pascal Hingamp ${ }^{12}$, Daniele Iudicone ${ }^{13}$, Olivier Jaillon $^{14,15,16}$, Stefanie Kandels-Lewis ${ }^{2,17}$, Lee Karp-Boss ${ }^{18}$, Eric Karsenti ${ }^{5,17}$, Uros Krzic ${ }^{19}$, Fabrice Not ${ }^{6,7}$, Hiroyuki Ogata $^{20}$, Stephane Pesant ${ }^{21,22}$, Jeroen Raes ${ }^{23,24,25}$, Emmanuel G. Reynaud ${ }^{26}$, Christian Sardet ${ }^{27,28}$,

940 Mike Sieracki $^{29, \dagger}$, Sabrina Speich ${ }^{30} \star$, Lars Stemmann ${ }^{9,31}$, Matthew B. Sullivan ${ }^{32}$, Shinichi Sunagawa ${ }^{2}$, Didier Velayoudon ${ }^{33}$, Patrick Wincker ${ }^{14,15,16}$

${ }^{1}$ Department of Marine Biology and Oceanography Institute of Marine Science (ICM)-CSIC Pg. Marítim de la Barceloneta 37-49, Barcelona, E08003, Spain

${ }^{2}$ Structural and Computational Biology, European Molecular Biology Laboratory, Meyerhofstr. 1, 94569117 Heidelberg, Germany

${ }^{3}$ Max-Delbrück-Centre for Molecular Medicine, 13092 Berlin, Germany

${ }^{4}$ School of Marine Sciences, University of Maine, Orono, Maine, USA

${ }^{5}$ Ecole Normale Supérieure, PSL Research University, Institut de Biologie de 1'Ecole Normale Supérieure (IBENS), CNRS UMR 8197, INSERM U1024, 46 rue d'Ulm, F-75005 Paris, France

$950{ }^{6}$ CNRS, UMR 7144, Station Biologique de Roscoff, Place Georges Teissier, 29680 Roscoff, France

7 Sorbonne Universités, UPMC Univ Paris 06, UMR 7144, Station Biologique de Roscoff, Place Georges Teissier, 29680 Roscoff, France

${ }^{8}$ Dept of Earth, Atmospheric and Planetary Sciences, Massachusetts Institute of Technology,

Cambridge, Massachusetts, USA

$955{ }^{9}$ CNRS, UMR 7093, LOV, Observatoire Océanologique, F-06230 Villefranche-sur-mer, France

${ }^{10}$ CNRS UMR 7232, BIOM, Avenue du Fontaulé, 66650 Banyuls-sur-Mer, France

${ }^{11}$ Sorbonne Universités Paris 06, OOB UPMC, Avenue du Fontaulé, 66650 Banyuls-sur-Mer, France

${ }^{12}$ Aix Marseille Université CNRS IGS UMR 725613288 Marseille, France

${ }^{13}$ Stazione Zoologica Anton Dohrn, Villa Comunale, 80121, Naples, Italy

$960{ }^{14}$ CEA - Institut de Génomique, GENOSCOPE, 2 rue Gaston Crémieux, 91057 Evry, France 
${ }^{15}$ CNRS, UMR 8030, CP5706, Evry, France

${ }^{16}$ Université d'Evry, UMR 8030, CP5706, Evry, France

${ }^{17}$ Directors' Research European Molecular Biology Laboratory Meyerhofstr. 169117 Heidelberg, Germany

${ }^{18}$ School of Marine Sciences, University of Maine, Orono, USA

${ }^{19}$ Cell Biology and Biophysics, European Molecular Biology Laboratory, Meyerhofstr. 1, 69117

Heidelberg, Germany

${ }^{20}$ Institute for Chemical Research, Kyoto University, Gokasho, Uji, Kyoto, 611-0011, Japan

${ }^{21}$ PANGAEA, Data Publisher for Earth and Environmental Science, University of Bremen, Bremen,

970 Germany

${ }^{22}$ MARUM, Center for Marine Environmental Sciences, University of Bremen, Bremen, Germany

${ }^{23}$ Department of Microbiology and Immunology, Rega Institute, KU Leuven, Herestraat 49, 3000

Leuven, Belgium

${ }^{24}$ Center for the Biology of Disease, VIB, Herestraat 49, 3000 Leuven, Belgium

$975{ }^{25}$ Department of Applied Biological Sciences, Vrije Universiteit Brussel, Pleinlaan 2, 1050 Brussels,

Belgium

${ }^{26}$ Earth Institute, University College Dublin, Dublin, Ireland

${ }^{27}$ CNRS, UMR 7009 Biodev, Observatoire Océanologique, F-06230 Villefranche-sur-mer, France

${ }^{28}$ Sorbonne Universités, UPMC Univ Paris 06, UMR 7009 Biodev, F-06230 Observatoire

980 Océanologique, Villefranche-sur-mer, France

${ }^{29}$ Bigelow Laboratory for Ocean Science, East Boothbay, Maine, USA

${ }^{30}$ Laboratoire de Physique des Océan UBO-IUEM Palce Copernic 29820 Polouzané, France

${ }^{31}$ Sorbonne Universités, UPMC Univ Paris 06, UMR 7093, LOV, Observatoire Océanologique, F-

06230, Villefranche-sur-mer, France

$985{ }^{32}$ Department of Ecology and Evolutionary Biology, Depts Molecular and Cellular Biology and Soil, Water and Environmental Science, University of Arizona, Tucson, Arizona, 85721, USA

${ }^{33}$ DVIP Consulting, 92310, Sèvres, France

$\dagger$ Current address: National Science Foundation, Arlington, Virginia, USA

$\$$ Current address: Department of Geosciences, Laboratoire de Météorologie Dynamique (LMD), Ecole

990 Normale Supérieure, 24 rue Lhomond 75231 Paris, Cedex 05, France

\section{Author Contributions}

S.R., and M.B.S. designed the study. C.D., M.P., and Sa.S., contributed extensively to sampling collection. S.K-L. managed the logistic of the Tara Oceans project. B.T.P., N.S. and E.L. performed the viral-specific processing of the samples. J.P., C.C., A.A., and P.W. lead the sequencing of viral samples. S.R., S.S. and B.E.D. lead the assembly of raw data. S.R., S.S., M.B.D. and M.B.S. analyzed the genomic diversity data. S.R., A.L., J.R.B. and M.B.S. analyzed the AMGs data. S.R., J.R.B., B.E.D, S.S., M.B.D., A.L., S.P., P.B., S.G.A., C.D., J.M.G., D.V. and M.B.S. provided constructive comments, revised and edited the manuscript. Tara Oceans coordinators provided creative environment and

1000 constructive criticism throughout the study. All authors discussed the results and commented on the manuscript.

\section{Author Information}

1005 All raw data are available at ENA or IMG, with sample identifiers indicated in Supplementary Table 1. Processed data, including assembled contigs, populations definition and abundance, clusters definition and abundance, and all annotated viral contigs are available at iVirus, http://mirrors.iplantcollaborative.org/browse/iplant/home/shared/ivirus. All scripts are available at MAVERICLab bitbucket: http://bitbucket.org/MAVERICLab/gov ecogenomics/overview. Reprints 
1010 and permissions information is available at www.nature.com/reprints. Correspondence and requests for materials should be addressed to mbsulli@gmail.com.

\section{References}

1. Falkowski, P. G., Fenchel, T. \& Delong, E. F. The Microbial Engines That Drive Earth's 1015 Biogeochemical Cycles. Science 320, 1034-9 (2008).

2. Suttle, C. A. Marine viruses--major players in the global ecosystem. Nat. Rev. Microbiol. 5, 801-812 (2007).

3. Rohwer, F. \& Thurber, R. V. Viruses manipulate the marine environment. Nature 459, 207-212 (2009).

1020 4. Breitbart, M. Marine Viruses: Truth or Dare. Ann. Rev. Mar. Sci. 4, 425-448 (2012).

5. Brum, J. R. \& Sullivan, M. B. Rising to the challenge: accelerated pace of discovery transforms marine virology. Nat. Rev. Microbiol. 13, 147-59 (2015).

6. Karsenti, E. et al. A holistic approach to marine eco-systems biology. PLoS Biol. 9, e1001177 (2011).

7. Duarte, C. M. Seafaring in the 21st Century : The Malaspina 2010 Circumnavigation Expedition.

1025 ASLO 11-14 (2015).

8. Lima-Mendez, G., Van Helden, J., Toussaint, A. \& Leplae, R. Reticulate representation of evolutionary and functional relationships between phage genomes. Mol. Biol. Evol. 25, 762-77 (2008).

9. Roux, S., Hallam, S. J., Woyke, T. \& Sullivan, M. B. Viral dark matter and virus-host interactions 1030 resolved from publicly available microbial genomes. Elife 4, 1-20 (2015).

10. Brum, J. et al. Patterns and ecological drivers of ocean viral communities. Science 348, 12614981-10 (2015).

11. Rosario, K. \& Breitbart, M. Exploring the viral world through metagenomics. Curr. Opin. Virol. 1, 289-97 (2011).

1035 12. Mizuno, C. M., Rodriguez-Valera, F., Kimes, N. E. \& Ghai, R. Expanding the marine virosphere using metagenomics. PLoS Genet. 9, e1003987 (2013).

13. Chow, C.-E. T., Winget, D. M., White, R. a., Hallam, S. J. \& Suttle, C. a. Combining genomic sequencing methods to explore viral diversity and reveal potential virus-host interactions. Front. Microbiol. 6, 1-15 (2015).

1040 14. Roux, S. et al. Ecology and evolution of viruses infecting uncultivated SUP05 bacteria as revealed by single-cell- and meta- genomics. Elife 3, 1-20 (2014).

15. Dutilh, B. E. et al. A highly abundant bacteriophage discovered in the unknown sequences of human faecal metagenomes. Nat. Commun. 5, 1-11 (2014).

16. Albertsen, M. et al. Genome sequences of rare, uncultured bacteria obtained by differential 
1045 coverage binning of multiple metagenomes. Nat. Biotechnol. 31, 533-8 (2013).

17. Sullivan, M. B. et al. Genomic analysis of oceanic cyanobacterial myoviruses compared with T4like myoviruses from diverse hosts and environments. Environ. Microbiol. 12, 3035-56 (2010).

18. Zhao, Y. et al. Abundant SAR11 viruses in the ocean. Nature 494, 357-360 (2013).

19. Labrie, S. J. et al. Genomes of marine cyanopodoviruses reveal multiple origins of diversity. Environ. Microbiol. 15, 1356-76 (2013).

20. Andersson, A. F. \& Banfield, J. F. Virus population dynamics and acquired virus resistance in natural microbial communities. Science 320, 1047-50 (2008).

21. Sunagawa, S. et al. Structure and function of the global ocean microbiome. Science 348, 1-10 (2015).

1055 22. Breitbart, M. \& Rohwer, F. Here a virus, there a virus, everywhere the same virus? Trends Microbiol. 13, 278-284 (2005).

23. Flores, C. O., Valverde, S. \& Weitz, J. S. Multi-scale structure and geographic drivers of crossinfection within marine bacteria and phages. ISME J. 7, 520-32 (2013).

24. Hurwitz, B. L., Brum, J. R. \& Sullivan, M. B. Depth-stratified functional and taxonomic niche specialization in the "core" and "flexible” Pacific Ocean Virome. ISME J. 9, 472-84 (2015).

25. Thompson, L. R. et al. Phage auxiliary metabolic genes and the redirection of cyanobacterial host carbon metabolism. Proc. Natl. Acad. Sci. U. S. A. 108, E757-64 (2011).

26. Dammeyer, T., Bagby, S. C., Sullivan, M. B., Chisholm, S. W. \& Frankenberg-Dinkel, N. Efficient phage-mediated pigment biosynthesis in oceanic cyanobacteria. Curr. Biol. 18, 442-8 (2008).

1065 27. Lindell, D., Jaffe, J. D., Johnson, Z. I., Church, G. M. \& Chisholm, S. W. Photosynthesis genes in marine viruses yield proteins during host infection. Nature 438, 86-9 (2005).

28. Sullivan, M. B. et al. Prevalence and Evolution of Core Photosystem II Genes in Marine Cyanobacterial Viruses and Their Hosts. PLoS Biol. 4, e234 (2006).

29. Anantharaman, K. et al. Sulfur Oxidation Genes in Diverse Deep-Sea Viruses. Science 344, 7571070760 (2014).

30. Friedrich, C. G., Bardischewsky, F., Rother, D., Quentmeier, A. \& Fischer, J. Prokaryotic sulfur oxidation. Curr. Opin. Microbiol. 8, 253-9 (2005).

31. Ghosh, W. \& Dam, B. Biochemistry and molecular biology of lithotrophic sulfur oxidation by taxonomically and ecologically diverse bacteria and archaea. FEMS Microbiol. Rev. 33, 9991075 (2009).

32. Santos, A. A. et al. A protein trisulfide couples dissimilatory sulfate reduction to energy conservation. Science 350, 1541-1546 (2015). 
33. Venceslau, S. S., Stockdreher, Y., Dahl, C. \& Pereira, I. A. C. The “bacterial heterodisulfide” DsrC is a key protein in dissimilatory sulfur metabolism. Biochim. Biophys. Acta 1837, 1148-64

35. Huergo, L. F., Chandra, G. \& Merrick, M. P(II) signal transduction proteins: nitrogen regulation

34. Dahl, C., Franz, B., Hensen, D., Kesselheim, A. \& Zigann, R. Sulfite oxidation in the purple sulfur bacterium Allochromatium vinosum: identification of SoeABC as a major player and relevance of SoxYZ in the process. Microbiology 159, 2626-38 (2013).

36. Leigh, J. A. \& Dodsworth, J. A. Nitrogen regulation in bacteria and archaea. Annu. Rev. Microbiol. 61, 349-77 (2007).

37. Pester, M., Schleper, C. \& Wagner, M. The Thaumarchaeota: an emerging view of their phylogeny and ecophysiology. Curr. Opin. Microbiol. 14, 300-6 (2011).

1090 38. Stahl, D. A. \& de la Torre, J. R. Physiology and diversity of ammonia-oxidizing archaea. Annu. Rev. Microbiol. 66, 83-101 (2012).

39. Lindell, D. et al. Genome-wide expression dynamics of a marine virus and host reveal features of co-evolution. Nature 449, 83-6 (2007).

40. Loy, A. et al. Reverse dissimilatory sulfite reductase as phylogenetic marker for a subgroup of sulfur-oxidizing prokaryotes. Environ. Microbiol. 11, 289-99 (2009).

41. Sabehi, G. et al. New insights into metabolic properties of marine bacteria encoding proteorhodopsins. PLoS Biol. 3, e273 (2005).

42. Weitz, J. S. et al. A multitrophic model to quantify the effects of marine viruses on microbial food webs and ecosystem processes. ISME J. 9, 1352-1364 (2015).

1100 43. Hamblin, S. R., White, P. a. \& Tanaka, M. M. Viral niche construction alters hosts and ecosystems at multiple scales. Trends Ecol. Evol. 1-6 (2014). doi:10.1016/j.tree.2014.08.005

44. Dennehy, J. J. What Ecologists Can Tell Virologists. Annu. Rev. Microbiol. 117-35 (2014). doi:10.1146/annurev-micro-091313-103436

45. Arcondéguy, T., Jack, R. \& Merrick, M. P II Signal Transduction Proteins, Pivotal Players in Microbial Nitrogen Control. Microbiol. Mol. Biol. Rev. 65, 80-105 (2001).

46. Pesant, S. et al. Open science resources for the discovery and analysis of Tara Oceans data. Sci. Data 2, 150023 (2015).

47. John, S. G. et al. A simple and efficient method for concentration of ocean viruses by chemical flocculation. Environ. Microbiol. Rep. 3, 195-202 (2011).

1110 48. Hurwitz, B. L., Deng, L., Poulos, B. T. \& Sullivan, M. B. Evaluation of methods to concentrate and purify ocean virus communities through comparative, replicated metagenomics. Environ. 
Microbiol. 15, 1428 - 1440 (2012).

49. Aminot, A., Kérouel, R. \& Coverly, S. in Pract. Guidel. Anal. Seawater 143-176 (2009).

50. Tara Oceans Consortium, C. \& Tara Oceans Expedition, P. Registry of all samples from the Tara Oceans Expedition (2009-2013). (2015). doi:10.1594/PANGAEA.842197

51. Tara Oceans Consortium, C. \& Tara Oceans Expedition, P. Environmental context of all samples from the Tara Oceans Expedition (2009-2013). (2015). doi:10.1594/PANGAEA.853810

52. Tara Oceans Consortium, C. \& Tara Oceans Expedition, P. Biodiversity context of all samples from the Tara Oceans Expedition (2009-2013). (2015). doi:10.1594/PANGAEA.853809

1120 53. Salazar, G. et al. Global diversity and biogeography of deep-sea pelagic prokaryotes. ISME J. 1-13 (2015). doi:10.1038/ismej.2015.137

54. Kultima, J. R. et al. MOCAT: a metagenomics assembly and gene prediction toolkit. PLoS One 7, e47656 (2012).

55. Peng, Y., Leung, H. C. M., Yiu, S. M. \& Chin, F. Y. L. IDBA-UD: a de novo assembler for single-

1125 cell and metagenomic sequencing data with highly uneven depth. Bioinformatics 28, 1420-1428 (2012).

56. Langmead, B. \& Salzberg, S. L. Fast gapped-read alignment with Bowtie 2. Nat. Methods 9, 357-9 (2012).

57. Luo, R. et al. SOAPdenovo2 : an empirically improved memory-efficient short-read de novo $1130 \quad$ assembler. Gigascience 1, 1-6 (2012).

58. Li, W. \& Godzik, A. Cd-hit: a fast program for clustering and comparing large sets of protein or nucleotide sequences. Bioinformatics 22, 1658-9 (2006).

59. Kang, D. D., Froula, J., Egan, R. \& Wang, Z. MetaBAT, an efficient tool for accurately reconstructing single genomes from complex microbial communities. PeerJ 3, e1165 (2015).

1135 60. Mavromatis, K., Ivanova, N., Barry, K. \& Shapiro, H. Use of simulated data sets to evaluate the fidelity of metagenomic processing methods. Nat. Methods 4, 495-500 (2007).

61. Roux, S., Enault, F., Hurwitz, B. L. \& Sullivan, M. B. VirSorter: mining viral signal from microbial genomic data. PeerJ 3, e985 (2015).

62. Pope, W. H. et al. Whole genome comparison of a large collection of mycobacteriophages reveals a continuum of phage genetic diversity. Elife 4, (2015).

63. Enright, A. J., Van Dongen, S. \& Ouzounis, C. A. An efficient algorithm for large-scale detection of protein families. Nucleic Acids Res. 30, 1575-84 (2002).

64. Finn, R. D. et al. Pfam: the protein families database. Nucleic Acids Res. 42, D222-30 (2014).

65. Eddy, S. R. Accelerated Profile HMM Searches. PLoS Comput. Biol. 7, e1002195 (2011). 
1145 66. Brum, J. R. et al. Illuminating structural proteins in viral “dark matter” with metaproteomics. Proc. Natl. Acad. Sci. U. S. A. 113, (2016).

67. Holmfeldt, K. et al. Twelve previously unknown phage genera are ubiquitous in the global oceans. Proc. Natl. Acad. Sci. U. S. A. 110, 12798-12803 (2013).

68. Kang, I., Jang, H. \& Cho, J.-C. Complete genome sequences of two Persicivirga bacteriophages, P12024S and P12024L. J. Virol. 86, 8907-8 (2012).

69. Kang, I., Oh, H.-M., Kang, D. \& Cho, J.-C. Genome of a SAR116 bacteriophage shows the prevalence of this phage type in the oceans. Proc. Natl. Acad. Sci. U. S. A. 110, 12343-8 (2013).

70. Hjorleifsdottir, S., Aevarsson, A., Hreggvidsson, G. O., Fridjonsson, O. H. \& Kristjansson, J. K. Isolation, growth and genome of the Rhodothermus RM378 thermophilic bacteriophage. Extremophiles 18, 261-70 (2014).

71. Marks, T. J. \& Hamilton, P. T. Characterization of a thermophilic bacteriophage of Geobacillus kaustophilus. Arch. Virol. 159, 2771-5 (2014).

72. Halmillawewa, A. P., Restrepo-Córdoba, M., Yost, C. K. \& Hynes, M. F. Genomic and phenotypic characterization of Rhizobium gallicum phage vB_RglS_P106B. Microbiology 161, 611-20 (2015).

73. Rohwer, F. \& Edwards, R. The Phage Proteomic Tree : a Genome-Based Taxonomy for Phage. J. Bacteriol. 184, 4529-4535 (2002).

74. Letunic, I. \& Bork, P. Interactive Tree Of Life (iTOL): an online tool for phylogenetic tree display and annotation. Bioinformatics 23, 127-128 (2007).

1165 75. Letunic, I. \& Bork, P. Interactive Tree Of Life v2: online annotation and display of phylogenetic trees made easy. Nucleic Acids Res. 39, W475-8 (2011).

76. Edwards, R. A., McNair, K., Faust, K., Raes, J. \& Dutilh, B. E. Computational approaches to predict bacteriophage-host relationships. FEMS Microbiol. Rev. in press, (2015).

77. Bland, C. et al. CRISPR recognition tool (CRT): a tool for automatic detection of clustered regularly interspaced palindromic repeats. BMC Bioinformatics 8, 209 (2007).

78. Rho, M., Wu, Y.-W., Tang, H., Doak, T. G. \& Ye, Y. Diverse CRISPRs Evolving in Human Microbiomes. PLoS Genet. 8, e1002441 (2012).

79. Rice, P., Longden, I. \& Bleasby, a. EMBOSS: the European Molecular Biology Open Software Suite. Trends Genet. 16, 276-7 (2000).

1175 80. Marçais, G. \& Kingsford, C. A fast, lock-free approach for efficient parallel counting of occurrences of k-mers. Bioinformatics 27, 764-70 (2011).

81. Oksanen, J. et al. The vegan Package. (2008).

82. Sharon, I. et al. Comparative metagenomics of microbial traits within oceanic viral communities. 
1180 83. Edgar, R. C. MUSCLE: a multiple sequence alignment method with reduced time and space complexity. BMC Bioinformatics 5, 113 (2004).

84. Waterhouse, A. M., Procter, J. B., Martin, D. M. A., Clamp, M. \& Barton, G. J. Jalview Version 2--a multiple sequence alignment editor and analysis workbench. Bioinformatics 25, 1189-1191 (2009).

1185 85. Price, M. N., Dehal, P. S. \& Arkin, A. P. FastTree 2--approximately maximum-likelihood trees for large alignments. PLoS One 5, e9490 (2010).

86. Huelsenbeck, J. P. \& Ronquist, F. MRBAYES: Bayesian inference of phylogenetic trees. Bioinformatics 17, 754-755 (2001).

87. Schliep, K. P. phangorn: phylogenetic analysis in R. Bioinformatics 27, 592-3 (2011).

1190 88. Sullivan, M. J., Petty, N. K. \& Beatson, S. A. Easyfig: a genome comparison visualizer. Bioinformatics 27, 1009-10 (2011).

89. Roy, A., Kucukural, A. \& Zhang, Y. I-TASSER: a unified platform for automated protein structure and function prediction. Nat. Protoc. 5, 725-38 (2010).

90. Wiederstein, M. \& Sippl, M. J. ProSA-web: interactive web service for the recognition of errors in three-dimensional structures of proteins. Nucleic Acids Res. 35, W407-10 (2007).

91. Schloissnig, S. et al. Genomic variation landscape of the human gut microbiome. Nature 493, 4550 (2013).

92. Alberti, A. et al. Comparison of library preparation methods reveals their impact on interpretation of metatranscriptomic data. BMC Genomics 15, 1-13 (2014).

1200 93. Longhurst, A. Ecological geography of the sea. (2007).

94. Krogh, A., Larsson, B., von Heijne, G. \& Sonnhammer, E. L. L. Predicting transmembrane protein topology with a hidden Markov model: application to complete genomes. J. Mol. Biol. 305, 56780 (2001). 